\title{
Lecturas y cuestionamientos al plano topográfico de la ciudad de Mérida 1864-1867. Solitario devenir en la construcción de una historia local
}

\author{
Gladys Noemi Arana López*
}

El objetivo de este trabajo es realizar una interpretación al plano de la ciudad de Mérida conocido como el Salazar llarregui, efectuado en los tiempos del Segundo Imperio en la capital del Estado de Yucatán en la república mexicana. Se parte del supuesto de que, si bien el plano es una representación de la ciudad de aquel entonces con información necesaria y de utilidad para las nuevas autoridades, el documento también tuvo como objetivo servir de soporte y contexto para la caracterización ideal de un emplazamiento, así como para plasmar los múltiples proyectos a ejecutar por la recién instalada administración. El planteamiento teórico-metodológico parte de la propuesta realizada y desarrollada por Priscilla Connolly para el análisis del plano Forma y Levantado de la Ciudad de México 1628 de Juan Gómez de Trasmonte (Connolly, 2008, pp. 116-134) de donde se derivan:

I. El mapa (plano) es el territorio, como representación de un territorio de acuerdo con las convenciones de las ciencias geográficas.

II. El mapa (plano) no es el territorio, sino una imagen en papel que representa aspectos seleccionados de un territorio mediante simbologías visuales codificadas culturalmente.

III. Los territorios son mapas (planos), por lo que complementariamente al anterior, debemos entonces conceptuar que el territorio debe de ser conocido y comprendido exclusivamente a partir de lo que está representado.

Para poder desarrollar esta propuesta, se realizaron análisis a varios documentos, obteniendo de ellos información de gran utilidad para la construcción de diversos contextos, que posibilitaron la puesta en valor del plano, de su autor, así como de la información contenida en él. Entre las fuentes estudiadas se encuentran los documentos redactados por la emperatriz Carlota en su viaje a Yucatán, las cartas de los gobernadores del Estado expresando sus necesidades o solicitando apoyo económico, militar o político al extranjero, algunos libros escritos por viajeros, los diarios de campo de José Salazar llarregui, planos de otras ciudades mexicanas, latinoamericanas y europeas, documentos legales del comisario imperial, multiplicidad de imágenes y algunos documentos escritos por investigadores locales en relación con la arquitectura y el urbanismo de la época.

* Cómo citar este artículo: Arana, G, N. (2013). Lecturas y cuestionamientos al plano topográfico de la ciudad de Mérida 1864-1867. Solitario devenir en la construcción de una historial local. En Apuntes 26 (1): 150 - 169. 


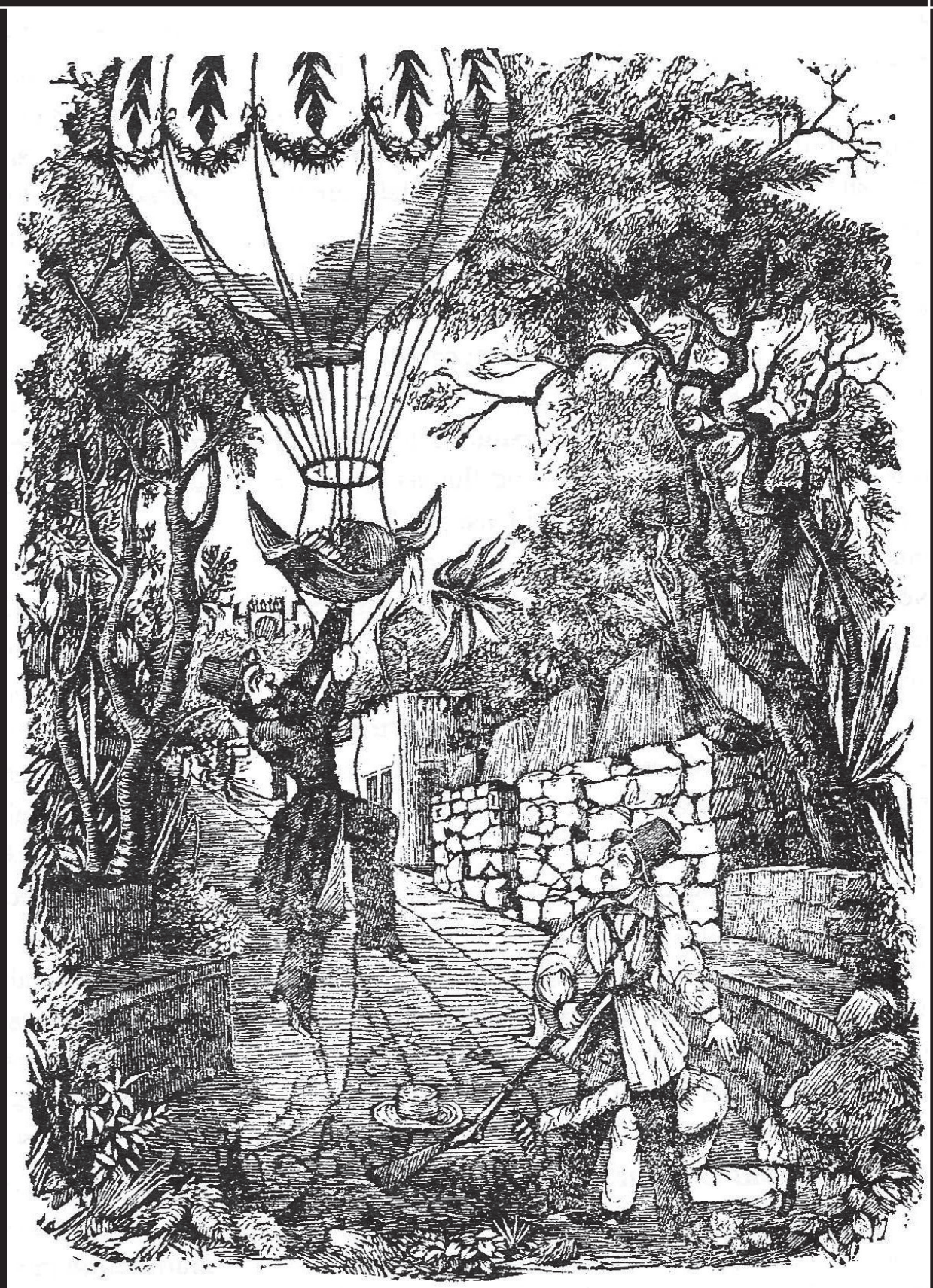

Imagen de la ciudad de Mérida.

Fuente:

D. Bullebulle, periódico burlesco y de

extravagancias redactado por una sociedad bulliciosa. 1847. Edición facsimilar, Mérida, Instituto de Cultura de Yucatán, 205, p. 135 


\section{Lecturas y cuestionamientos al plano topográfico de la ciudad de Mérida 1864-1867. Solitario devenir en la construcción de una historia local}

Readings and Questions Around the Topographic Map of Merida

1864-1867. Solitary Construction of a Local History

Leitura e questionamiento do mapa topográfico da cidade de Mérida 1864-

1867. Tornando-se a contrução solitaria de um história local

Gladys Noemí Arana López

gladys.arana@gmail.com

Arquitecta de la Universidad Autónoma de Yucatán. Magíster en Técnicas de Energías Renovables de la UIA, Huelva, España (Mención Sobresaliente por Unanimidad), Magíster en Arquitectura de la FAUADY y Doctora en Arquitectura de la Universidad Michoacana de San Nicolás de Hidalgo en Morelia, Michoacán, (Mención honorífica). Profesora de carrera de tiempo completo en la Facultad de Arquitectura de la Universidad Autónoma de Yucatán en donde imparte materias relacionadas con teoría, crítica e historia de la arquitectura.

Universidad Autónoma de Yucatán

Resumen

El objetivo principal de este documento fue realizar la lectura de un plano topográfico del siglo XIX, considerando que: a) El mapa es el territorio, lo que implica que es una representación realizada de acuerdo a convencionalismos propios de las ciencias geográficas de la época en cuestión; b) El mapa no es el territorio, sino únicamente una imagen de él en el papel, en donde se observan diversas series de símbolos y códigos culturales; y por último c) El territorio es el mapa y por consiguiente debe ser conocido a partir de lo que está representado. El ejercicio se realizó con el plano conocido en la región como el Salazar llarregui y cuyo nombre oficial es Plano topográfico de la Ciudad de Mérida. Este es el primer plano de la ciudad del que se tiene notica, realizado en los tiempos del imperio de Maximiliano de Habsburgo. Para la comprensión integral de este documento, se recurrió a diversas fuentes documentales y gráficas, lo que permitió contrastar algunas aseveraciones realizadas con anterioridad, así como plantear nuevas interrogantes con relación al documento. La continuidad de este trabajo presenta muchas oportunidades, ya que a partir de él se pueden estudiar las dinámicas de crecimiento urbano, las densidades, los patrones arquitectónicos, así como las preexistencias y permanencias arquitectónicas o urbanas, entre otras tantas posibilidades.

Palabras clave: Plano, ciudad histórica, zona urbana, historia nacional mexicana, arquitectura

Descriptores: Desarrollo urbano-Historia- México, arquitectura mexicana-Aspectos sociales, historiografía

Abstract

The main objective of this paper was to make a reading of a nineteenth century topographic map, considering that: a) The map is the territory, implying that a representation is made according to its own conventions of geosciences at the time in question; b) The map is not the territory, but only a picture of it in paper, where there are different sets of cultural codes and symbols; and finally, c) The territory is the map (plan) and therefore should be known from what is

Artículo de reflexión represented. The study took place in the plan of the region known as the Salazar llarregui, officially called Plano topográfico de la Ciudad de Mérida. ,This is the first known plan of the city, drawn at the time of Maximilian of Hapsburg's empire. To truly understand this plan, a mix of documentary sources and graphs were studied. This helped to contrast some assertions made previously, and to raise new questions regarding the document. The continuity of this work presents many opportunities, since from it you can study the dynamics of urban growth, densities, architectural patterns and pre-existing architectural or urban continuities in relation to the present, among many other possibilities .

Keywords: plan, historic city, urban, Mexican national history, architecture

Keywords plus: Urban development-History-Mexico, Mexican architecture-Social, historiography

fundamentación de la tesis doctoral "La vivienda de la burguesía en Mérida al cambio de siglo (18861916). La vida cotidiana en el ámbito privado" que se presentó en marzo de

2011 y obtuvo Mención Honorífica en la Universidad Michoacana de San Nicolás de Hidalgo, en Morelia, Michoacán, México. La dirección de la tesis estuvo a cargo de la Dra. en Arq.

Catherine Ettinger.

Resumo

O objetivo principal deste trabalho era fazer a leitura de um mapa topográfico do século XIX, aproximando-se sob três pontos de vista diferentes, mas complementares: a. o mapa é o território, o que implica que a representação é feita de acordo com suas próprias convenções de geociências na época em questão, b. o mapa não é o território, mas apenas uma imagem dele no jornal, onde há diferentes conjuntos de códigos e símbolos culturais e, finalmente, c. o território é o mapa (plano) e, portanto, deve ser conhecido a partir do que é representado. Especificamente, o estudo foi realizado fora do avião na região conhecida como o llarregui Salazar e oficialmente chamado de mapa topográfico da cidade de Mérida, a primeira cidade conhecida e realizada no momento do império de Maximiliano de Habsburgo. Para compreensão abrangente do presente trabalho utilizou uma combinação de fontes documentais e gráficos, e pode contrastar algumas afirmações feitas anteriormente, e levantar novas questões sobre o documento. A continuidade deste trabalho apresenta muitas oportunidades, já que a partir dele você pode estudar a dinâmica de crescimento urbano, densidades, padrões arquitetônicos e pré-existentes continuidades arquitetônicas ou urbano e em relação ao presente, entre muitas outras possibilidades.

Palabras-chave: plano, cidade histórica, urbano, história nacional mexicana, arquitetura

Palavras-chave descritores: Desenvolvimento Urbano-História-México, México arquitetura social, a historiografia

doi:10.11144/Javeriana.APC26-1.Icpt

* Los descriptores y keywords plus están normalizados por la Biblioteca General de la Pontificia Universidad Javeriana. 
Los documentos enunciados previamente, se organizaron a manera de una gran serie documental compuesta por otras mucho más pequeñas, complementarias entre sí. Con ellas se realizaron ejercicios de confrontación y lecturas transversales de información, con la intención de ratificar o invalidar dialógicamente los resultados obtenidos en una primera lectura. Esto posibilitó responder algunas de las siguientes preguntas que se platearon de forma exclusiva con relación al plano de Mérida de 1864: ¿Qué dice el plano sobre el territorio? ¿El plano representa la ciudad ideal o la real? ¿Es un proyecto de modernización?, o bien ¿se integran en él, lo real, lo ideal y lo posible? ¿Cómo se relaciona la técnica de representación con la verdadera intención? ¿Qué se omite en el plano? ¿Cuál fue el criterio de selección de los edificios enunciados en este? ¿El plano era parte de la construcción de un nuevo Estado nacional? ¿Qué difusión e impacto tuvo?

El plano ha sido estudiado tangencialmente, ya que se le ha empleado como fuente de información y documento base para la interpretación de la nomenclatura de la Mérida imperial. ${ }^{1}$ Particularmente la propuesta de Aercel Espadas (1991, pp. 1-16) fue desarrollada a partir de la concepción teórica y metodológica propia para el estudio de la nomenclatura de las ciudades que según Espadas, debe ir más allá de las cuestiones anecdóticas o románticas, para poderlas aplicar a modo de fuente para el establecimiento de un método científico y sistemático de nomenclaturar (sic). En el trabajo de Marco Tulio Peraza (2009, pp. 129-144) se observa una descripción del documento a partir de la propuesta de la nomenclatura imperial y de algunas de las representaciones planimétricas de los edificios más importantes de la época, sin embargo el plano a fin de cuentas, es visto como un documento destinado al olvido al haber sido creado en el contexto de un modelo político caduco, incluso para su época. Otros trabajos emplean el plano como base o punto de partida para demostrar por medio de la contrastación, el crecimiento de la ciudad de Mérida o bien como contexto histórico general ${ }^{2}$.

Históricamente hablando, el documento en cuestión se realizó en un momento en el que México pasaba por momentos más que difíciles. Después de los intentos españoles por reconquistar el país, las invasiones norteamericanas, los interminables conflictos entre los liberales y los conservadores, el enojo de algunos sectores de la sociedad mexicana por la política del gobierno del presidente Juárez y las arcas nacionales completamente vacías, se le ofreció a Maximiliano de Austria la posibilidad de ser emperador de México. Él había llegado a México en 1864 y uno de sus objetivos era desarrollar económica y socialmente los territorios mexicanos bajo su custodia. Las labores para la realización del plano de la ciudad de Mérida se iniciaron recién había llegado a la ciudad José Salazar llarregui, nombrado comisario Imperial para la península.

En el plano topográfico de la ciudad de Mérida se registró toda la información obtenida a partir de un levantamiento realizado a nivel de piso (Espadas, 1993, p. 79), en el que se puede apreciar la organización de todas las manzanas que conformaban la ciudad en aquel entonces, así como los lotes, cada una de las construcciones y las zonas de cultivo localizadas en su periferia. El documento original a partir del cual se realizó este trabajo lo imprimió el lng. Enrique A. Cervantes en blanco y negro con una de las piedras litográficas originales en $1945^{3}$. Vale la pena señalar que si bien la piedra litográfica se trasladó a Mérida en 1866 con el objeto de imprimir ejemplares para vender al público en general ${ }^{4}$, aparentemente $\mathrm{y}$ al menos en acervos documentales públicos, no existe un plano original, únicamente una litografía del mismo en la Biblioteca Nacional de Francia ${ }^{5}$.

La estructura del presente documento está organizada de lo general a lo particulary de forma congruente con el planteamiento metodológico. En primera instancia se presenta un planteamiento contextual en el que se hace referencia a la situación socioeconómica y política que primaba en México y particularmente en Yucatán, con la intención de comprender las circunstancias de vida y de trabajo de quien dirigió la elaboración del plano, así como del documento en sí, considerando que tanto los ámbitos urbanos como los arquitectónicos representados derivan de estas circunstancias y situaciones. En segundo lugar se exponen las interpretaciones, tanto de lo representado como de lo faltante y de las posibilidades entre lo real y lo ideal; por último vienen las reflexiones y conclusiones al respecto.

No se pretende ser exahustivo en relación al documento en sí (las técnicas empleadas en su elaboración, los métodos del levantamiento, las vidas de quienes realizaron el trabajo) así como
1. Este plano es de importancia histórica en la región y en particular para Mérida, ya que se le considera el primer plano conocido de ella (Peraza, 2009, p. 129; Espadas, 2011, p. 8). El primero del que se tiene noticias, fue realizado por el arquitecto Sergio Servián en 1813 y se consideró extraviado desde 1820 (Antochiw y Alonzo,, 2010, pp. 173-178).

2. Entre estos se encuentra Blanca Paredes (2009). Mérida en el siglo XX. La lectura de su planimetría. En Vargas y Ayala (coords.). Arquitectura y Ciudad. Métodos Historiográficos: Análisis de Fuentes Gráficas. México: UAM-XOCH y Aercel Espadas (2011). Mérida sin $\mathrm{M}$ ni R., sin mitos ni romanticismos de su fundación hasta su segunda modernización. En Cuadernos de Arquitectura de Yucatán, entre algunos otros.

3. Localizado en la mapoteca Manuel Orozca y Berra, varilla CGYUC11, número de control 21265-cge-7264.

4. En locales populares de la época como lo eran la librería de Rodulfo G. Cantón y en la imprenta de José Dolores Espinoza, a 4 pesos el ejemplar marquilla y a 5 pesos en papel más grueso (Canto, E., 2006).

5. El documento que se encuentra en la Biblioteca Nacional de Francia está catalogado con la siguiente descripción: "Plano topografico de la Ciudad de Merida. Levantado con arreglo à las instrucciones del Exmo. Sr. Comisario Imperial de la Peninsula de Yucatan Jose Salazar llarregui por los Ingenieros de la Comision Cientifica que nombro: Mauricio von Hippel, Carlos Ramiro, Francisco de P. Beltran y Carlos Moya bajo la direccion del Gefe de la Seccion topografica Augustin Diaz. Escala à 1: 5000 Gravé par Regnier et Dourdet [Document cartographique]. Autor: Agustín Díaz. Tamaño del documento: 1050 por $860 \mathrm{cms}$. Impreso en Gratia et Schwaerzlé (Paris)". 
Figura 1.

Imagen cotidiana en la ciudad de Mérida.

Procesión en la calle 60.

Fuente:

Desiree Charnay, Viaje a Yucatán, 1860, p. 340.

6. Entre aquellos que han estudiado al Segundo Imperio, sus circunstancias o personajes, se encuentran Noäl Salomon, Ernesto de la Torre Villar, Jesús de León Toral, Daniel Moreno, Adelina Zendejas, Ernesto Tárrago, Enrique Florescano, Justo Sierra y José María Iglesias, por mencionar algunos. También se han escrito algunas novelas que se consideran como esenciales para comprender la época, entre ellas se encuentran Noticias del Imperio de Fernando del Paso y Península, Península de Hernán Lara Zavala, entre otras.

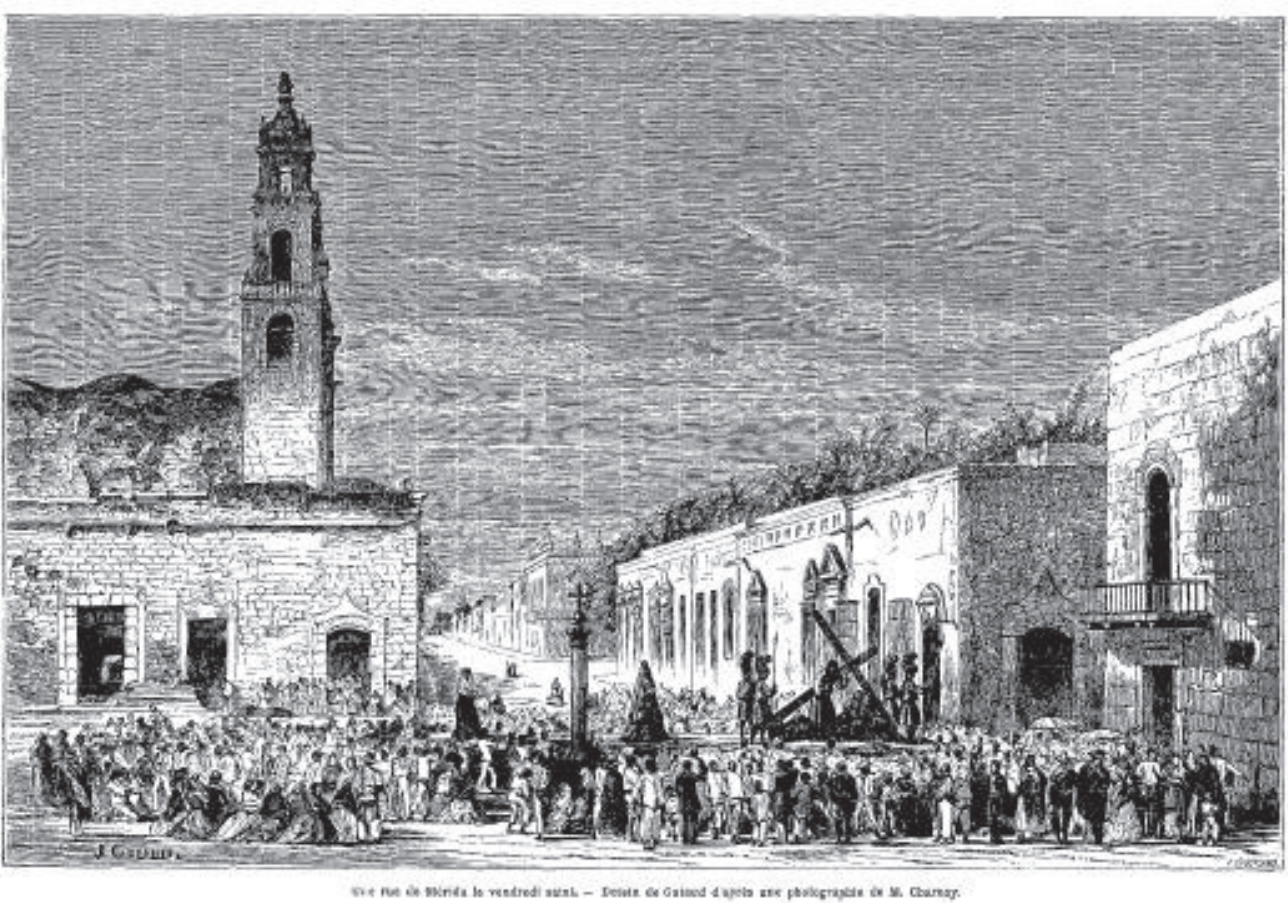

tampoco en relación a los edificios representados o aquellos que faltaron por representar. En realidad, se trata de plantear algunas preguntas e inquietudes sobre cuestiones que siempre se han dado por ciertas y a la vez, otorgarle al plano una nueva dimensión que va mucho más allá de sus límites físicos.

El hombre y su tiempo. José Salazar llarregui y sus múltiples encomiendas

Los hechos que posibilitaron que Maximiliano de Habsburgo se volviera emperador de México y Salazar llarregui comisario Imperial en la península de Yucatán fueron muchos y muy complejos ${ }^{6} \mathrm{y}$ es que después de cerca de cuarenta años en los cuales México había vivido como nación independiente, no había logrado estabilizarse ni política, ni económica y mucho menos socialmente.

En el año de 1860, Benito Juárez en su calidad de presidente de la república mexicana, declaró la suspensión de pagos de la deuda externa, debido a la desafortunada situación financiera en la cual se encontraba el país, decisión que fue tomada en complementariedad con otras acciones en aras de "salvar a la sociedad y reorganizar nuestra hacienda para poder satisfacer más adelante nuestros compromisos...” (Monroy, 2003, 2)

Los principales países con deudas por cobrar o bien con intereses económicos en México eran Inglaterra, Francia y España que, aún habiendo expresado que no intervendrían o impondrían política alguna en el país, realmente tenían otras intenciones. Así, cada una de estas naciones pretendía algo en particular. España esperaba poder recuperar los territorios que años atrás habían sido suyos, Inglaterra buscaba eliminar la participación económica de los Estados Unidos sobre México y Latinoamérica, mientras Francia pretendía crear y consolidar un imperio franco-americano.

En 1864 Yucatán se enfrentó con un bloqueo francés. El enfrentamiento de los yucateros frente a los franceses fracasó y terminó con la recapitulación que reconocía al Segundo Imperio y se adhería al mismo. A partir de ese momento se inició la reversión de las leyes de Reforma y consecuentemente, todos los cambios que se habían efectuado en materia religiosa-institucional (Monroy, 2003, p. 9). Entre estas estaban la ley Juárez que suprimía los tribunales especiales y acotaba la jurisdicción de los eclesiásticos y militares; la ley Lerdo que ordenaba la desamortización de todas las propiedades rústicas y urbanas de las corporaciones eclesiásticas y civiles; la ley orgánica del Registro del Estado Civil que tenía como objetivo regular el establecimiento y uso de los cementerios; así como la ley lglesias que prohibía cobrar a los pobres obvenciones y derechos por bautismos, amonestaciones, casamientos y entierros (Quezada, 2011, p. 148).

Particularmente se suprimieron a los jueces del registro civil, devolviéndole su función a los 
curas y párrocos, se derogó la ley de instrucción pública, se suspendió la nacionalización de capitales en manos de la iglesia y la liberación de las hipotecas de las propiedades rurales (haciendas y ranchos) y urbanas y facultaron a los clérigos para realizar actos y prácticas religiosas en lugares públicos, sin permiso de la autoridad política (Quezada, 2011, pp. 154 155).

Yucatán prevalecía prácticamente por su cuenta, muy a pesar de la anexión a la nación mexicana concretada en 1848 (Lapointe, 2008, p. 27), ya que incluso en ese mismo año, mientras se libraba en la región la llamada Guerra de Castas, se realizó una solicitud de ayuda para concluir con ese movimiento, no al gobierno mexicano sino al presidente de los Estados Unidos, de la misma manera como se había hecho a España y a Gran Bretaña con el ofrecimiento de ser parte de cualquiera de estas naciones a cambio de ayuda (Quezada, 2011, p. 144).

The people of Yucatan cannot allow themselves to be murdered and destroyed without employing every means in their reach to avoid it. They must, therefore, appeal to some foreign power, invoking their favor, the rights of humanity and the sympathy which an enlightend and civilized people should extend to another of the same character?.

$\mathrm{Al}$ término de este conflicto armado $^{8}$, la región precisaba urgentemente una reordenación territorial, que incluía la revalorización de los lotes urbanos y rurales, así como la necesidad de una reconstrucción generalizada de la ciudad. (Quezada, 2011, p. 159). En Mérida, en aquel entonces vivían aproximadamente 26,068 personas (Suárez, 1977, p. 95). De las 123 manzanas que conformaban los primeros cuarteles de la ciudad únicamente cuatro docenas estaban densamente pobladas, siendo los barrios más consolidados los de San Juan y Santa Lucía. Por fuera de esta zona la ocupación del territorio era mínima (Vega, 2003, p. 100). Tanto la situación económica como el rezago material de la ciudad fueron duramente criticados y satirizados en diversas publicaciones de la época que reflejaban el sentir generalizado de la población:

Don Aniseto llevaba diez años de haber salido de Yucatán. (...) Yo le había escrito

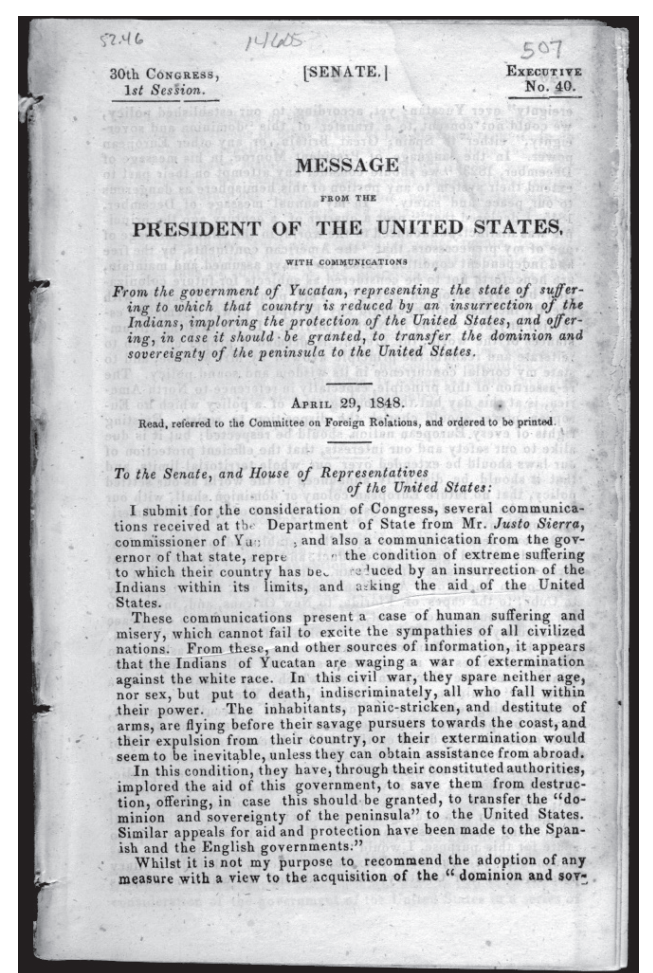

repetidas cartas manifestándole el desarrollo que desde el año de 1840 iba presentando por acá el espíritu de progreso, de mejoras y adelantos. Con esto se llenó de regocijo y entusiasmo, porque se había marchado con el desconsuelo de oír cantar a los viejos de su país. "Las cosas de Yucatán, déjalas como se están”. Y deseoso de palpar las reformas del suelo querido que le viera nacer y juguetear en ligeras andaderas y todo lo demás, hasta observar que saliese para el extranjero con algunas canas, tuvo la brillante idea de regresar a este país en un santiamén, lo que muy a su satisfacción llegó a conseguir. [...]

-¡Por Dios! ¡Pues mire que es un chasco! [...] ¿No han venido extranjeros a colonizar nuestros muchos terrenos incultos?

-No, señor [...]

- ¿Serán colegios, casas de enseñanza bien reglamentadas para la juventud de ambos sexos, sociedades literarias, nuevos ramos de industria, fábricas de telas y otros géneros, virtudes en los ciudadanos, patriotismo en los directores del pueblo?... ¿Qué, pues, es lo que hay? [...]

-Como todo son gastos y francachelas, es imposible que podamos dar un paso adelante. Si se ofrece hacer esto, aquello o
Figura 2.

Petición de anexión de Yucatán a Estados Unidos. Detalle de carta.

7. "United States. President and Polk, James K. Message from the President of the United States, With communications from the government of Yucatan, representing the state of suffering to which that country is reduced by an insurrection of the Indians, imploring the protection of the United States, and offering, in case it should be granted, to transfer the dominion and sovereignty of the peninsula to the United States". Government publications. 1848. Woodson Research Center, Rice University, Americas collection, 1811-1920, MS 518. http://hdl.handle. net/1911/27099, p 14.

8. Algunos autores mencionan el término de la misma después de ocho años de luchas, alrededor de 1855 , aunque oficialmente se declaró su conclusión en 1901 con la toma del poblado de Chan Santa Cruz (Reed, 1971, p. 5). 

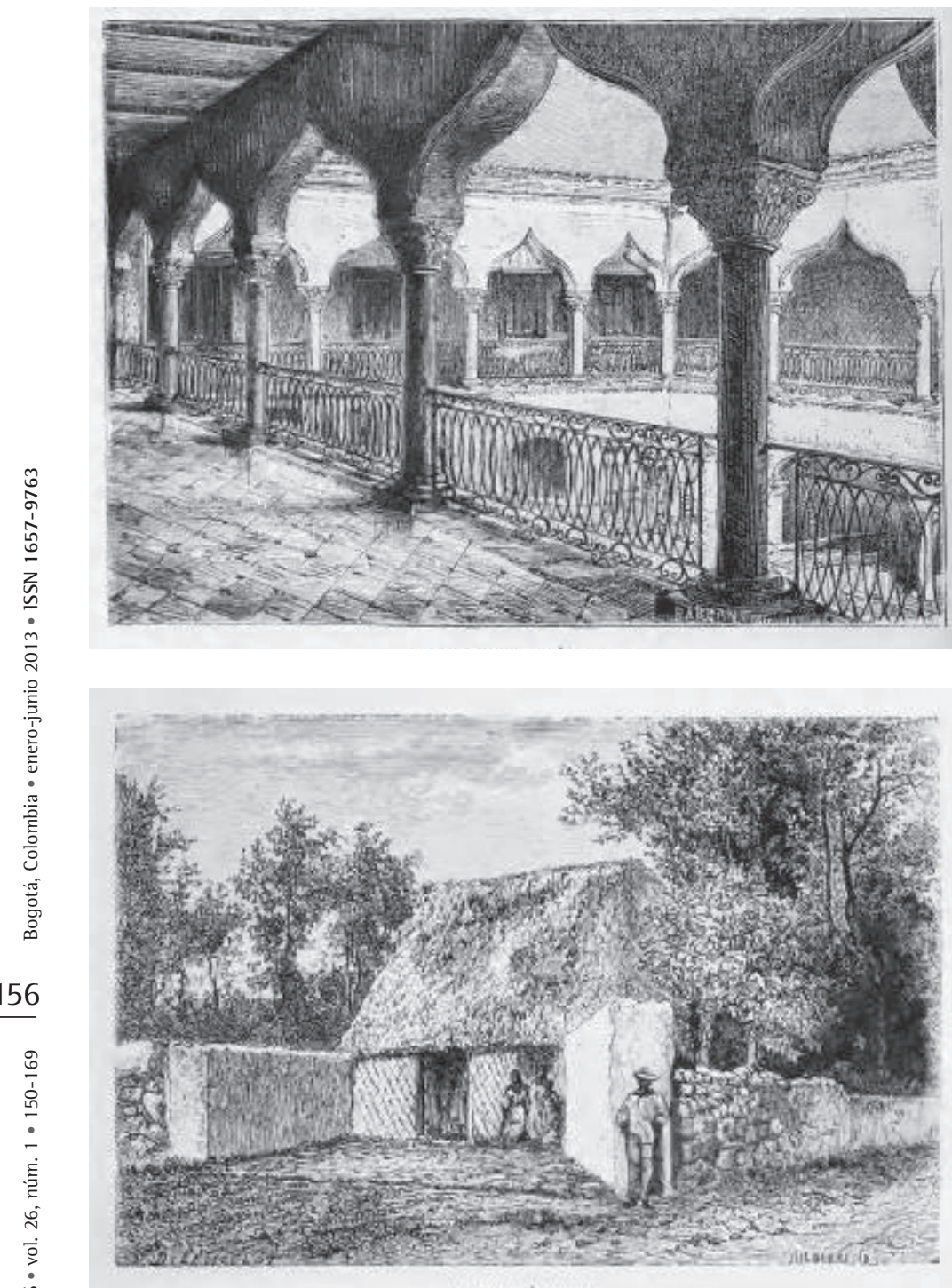

La vivienda no era muy diferente a aquella de los tiempos de la colonia. Todas las casas estaban alineadas a la calle y la mayoría tenían un patio central ortogonal alrededor del cual se organizaban las habitaciones. Los paramentos más densificados eran los orientados norte-sur con excepción de aquellas manzanas localizadas alrededor de alguna plaza de una iglesia.

Es así que en medio de disputas civiles, insurrecciones militares (Reed, 1971, p. 12) y falta de recursos económicos, el 3 de noviembre de ese mismo año desembarcó en el puerto de Sisal, el lng. José Salazar llarregui como Comisionado Imperial de Maximiliano de Habsburgo y junto con él, una Comisión Científica, un ejército organizado y dinero del gobierno imperial con el objetivo de volver a la península de Yucatán un posible centro político para los países de América central, ante el deseo que tenía Napoleón 111 de consolidar su imperio en esta área y mejorar las condiciones de vida en la península de Yucatán y en particular en Mérida.

\section{Mérida, noviembre de 1865}

Repito que en mi concepto es una persona muy digna contra la cual no se puede promover ninguna queja cualquiera de injusticia o faltas en el gobierno. Obra según su conciencia, tal vez en la sustancia de algunos decretos que dio hay errores o cosas que hubieran debido ser redactadas mejor, a fin de no chocar tanto, pero por otro lado es muy difícil, porque los yucatecos no quieren sino sus antiguas leyes y los menos gravámenes posibles, lo que vendría a parar en pagarles todo sin que contribuyeran ellos mismos para nada...(Iturriaga, 2011, p. 29).

Con la llegada del Segundo Imperio y de José Salazar llarregi como ejecutor de los deseos de Napoleón 111 y de Maximiliano de Habsburgo, una oleada de modernidad entró galopante a Mérida. Partidario incondicional y amigo personal del emperador, había sido parte de la corte de honor que viajó a Miramar a ofrecerle la corona de México y recibió a la pareja imperial en el puerto de Veracruz a solicitud del mismo Maximiliano. José Salazar realizó una administración que se caracterizó tanto por el apego a los lineamientos expresados por él, como a su capacidad pacificadora y conciliadora, al grado de que incluso 


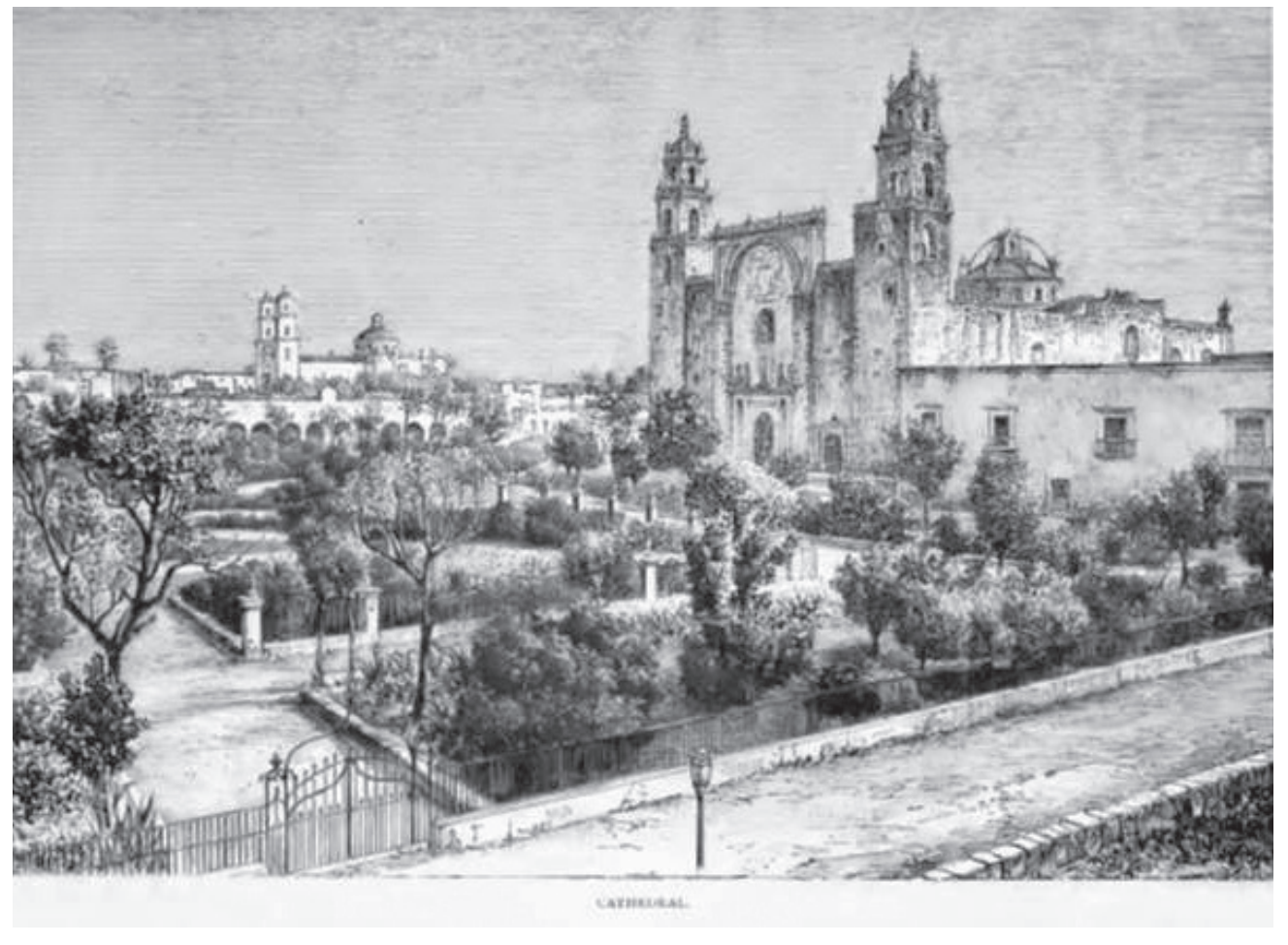

enemigos del incipiente emperador ,regresaron a estas tierras.

Don José Salazar Ilarregui was celebrated in Mexico as an astronomer, a civil engineer and a mining expert. He wrote a work on Astronomy and other text-books, and was made Chief of the Mexican Boundary Commission to locate the northern frontier in 1849 . He was again appointed to locate the line of the Gadsdsten purchase in 1854. When Maximilian and Carlota came to Mexico in 1864, Salazar was appointed to receive them at Veracruz.

The monarchs liked him so much that they became during the next three years on most intimate terms with him (...)

Imperial Commissary in Yucatan, Minister of State, Minister of the Interior, Minister of Fomento and again - at the enthusiastic request of the inhabitants - Commissary and General Commandant in Yucatan, Grand Cross of Guadalupe, - all these honours show their faith in Salazar and affection for him - and their faith was not misplaced. He served the Empire loyally to the last (...) A man of the strictest integrity, a scientist of no mean attainments, he lived honoured and died poor. January 4, 1892 (Hiersemann, 1913, pp. 26-27) ${ }^{9}$.
Nacido en Sonora en 1823, se graduó en ingeniería, geografía y astronomía, cursando sus estudios en el Colegio de Minería, institución en la que años después fue profesor y director. Nació y vivió en una época que debido a la tendencia racional del aprovechamiento de recursos, así como al enciclopedismo y al naturalismo, fue preciso dar a conocer riquezas hasta el momento ignoradas. Por otro lado, si bien el siglo XIX se caracterizó por los conflictos bélicos y por la inestabilidad política en México, fue un momento en el que se gestaron muchas instituciones académicas apoyadas tanto por liberales como por conservadores, republicanos y monárquicos, entre estas se encontraba la Sociedad Mexicana de Geografía y Estadística a la cual José Salazar llarregui pertenecía, por lo que es considerado como el heredero de una tradición iniciada por Carlos de Singuenza, Antonio de Alzate, Miguel Constanzó y Blas Blacácel (Pickenhayn, 2005, pp. 166-168).

Años antes de llegar a Mérida como representante imperial, Salazar había empezado a adquirir mucha experiencia sobre la agrimensura derivada de las múltiples encomiendas que había recibido en relación a esta actividad; como ejemplo de esto se encuentra el trabajo desarrollado entre los años 1849 y 1850, cuando perteneció a la Comisión de Límites mexicana, encargada de trazar las nuevas fronteras entre México y Estados Unidos derivadas del tratado de Guadalupe
Figura 6.

Ciudad de Mérida.

Panorámica de la

Plaza de Armas y

vista de la Catedral.

Fuente:

(1887). Ancient Cities of the New World, p. 273).

9. Catalogue Bibliotheca Mexicana, Karl W. Hiersemann, Leipzig, 1913. pp. 26-27, ítem 144. En el catálogo aquí referido, se incluye una colección importante de cartas, manuscritos e impresos que abarcan desde la conquista de México hasta el siglo XIX. En él se encuentra incluida la colección del Baron de Gerold-encargado de los negocios de Prusia en la ciudad de México desde 1840 hasta 1845 y amigo de Alexander von Humboldt. Constituida por 54 cartas firmadas por Maximiliano y nueve de Carlota, todas ellas de gran valor histórico. 


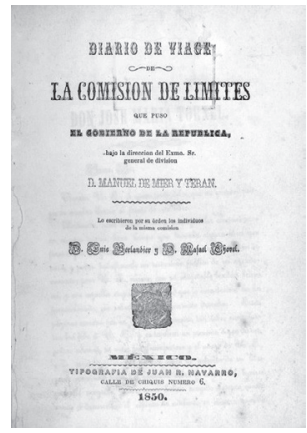

Figura 7.

Plano de la república mexicana.

Fuente:

Antonio García Cubas (1857). Atlas Geográfico, Estadístico e Histórico de la República Mexicana.

Figura 8.

Diario de Viaje de la Comisión de Límites. Portada.

Fuente:

http://hdl.handle. net/1911/27088.

10 La comisión estaba compuesta por el genera

D. Pedro García Conde, Comisario; ciudadano, José

Salazar llarregui, agrimen-

sor; Francisco Jiménez

y Francisco Martínez de Chavero como ingenieros de primera clase, además este último fue nombrado secretario de la comisión; Agustín

García Conde y Ricardo

Ramírez como ingenieros

de segunda clase, así como por Felipe de Iturbe como intérprete y traductor.

Salazar llarregui, José and International Boundary Commission (United States and Mexico) 1849-1853. Datos de los trabajos astronómicos y topográficos dispuestos en forma de diario: Practicados durante el año de 1849 y principios de 1850 por la Comision de limites Mexicana en la línea que divide esta republica de la de los EstadosUnidos, Practicados durante el año de 1849 y principios de 1850 por la Comisión de limites Mexicana en la línea que divide esta republica de la de los Estados-Unidos.

México: Imprenta de Juan R.

Navarro, 1850. From Woodson Research Center, Rice University. http://hdl.handle.net/1911/27088, p. 9

11 Revisión de la colección cartográfica de David Rumsey, consultable en http:// www.davidrumsey.com/, 15 de diciembre 2009 .

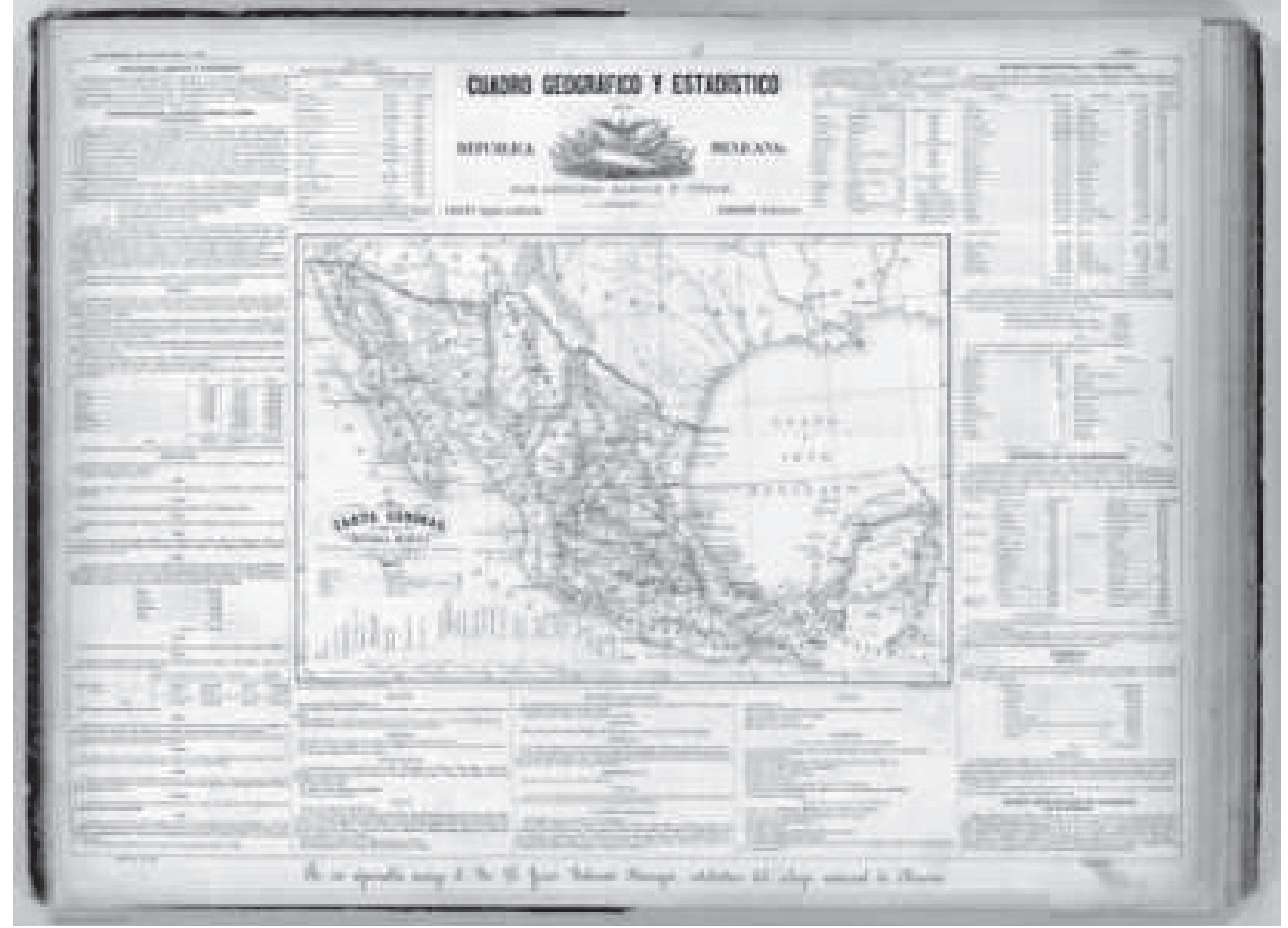

Hidalgo. La mencionada comisión estaba encabezada por el general D. Pedro García Conde y complementada con cuatro ingenieros auxiliares y un intérprete. La comisión norteamericana estaba encabezada por A.W. Whipple ${ }^{10}$.

En aquel entonces se estaban realizando muchos trabajos cartográficos en diversas ciudades latinoamericanas, europeas y americanas, como los de Joseph Meyer, en especial, con Grosse und vollstandiger Auswanderungs-Atlas fur Nordamerika y los de Tanner y Mitchell, conocido como Universal Atlas Maps of the Various U.S. States and Territories, Canada, and South America dated from 1844 to 1854 , que guardan una cercana similitud gráfica con el desarrollado en Mérida pocos años después ${ }^{11}$.

Yucatán, en poco más de un año, trabajó en la organización territorial, haciendo la división política del Departamento de Yucatán; en las comunicaciones, sobre todo mediante el tendido de la línea de telégrafo entre Mérida y Sisal; en la construcción de diversos caminos y vías férreas, también se creó el banco de avío, se fomentó la inmigración de colonos agricultores alemanes y la construcción de casas para ellos en Santa Elena Nohcacab (Durán-Merck, 2009, p. 10); se creó la figura de abogado de indios para su supuesta defensa y se instauró por decreto, el sistema métrico decimal. En Mérida se construyeron pozos artesianos y norias en distintos puntos de la ciudad, algunas calles se nivelaron, se instauró la policía y la vigilancia de las obras públicas, se creó una red de alumbrado público por medio de aceite de carbón y se pusieron rejas perimetrales a la capilla de Santiago y a la catedral -la cual recuperó su escudo-. De igual forma, se inaguró el Museo Yucateco, se realizaron litografías de las iglesias de Mérida y se publicó el libro Due küstenbildung des nördliche Yukatan (Quezada, 2011, p. 155; Espadas, 1991, p. 3).

Recomendaciones de la emperatriz Carlota a los comisarios

México, octubre de 1865?

Lo que es necesario...

Escuchar a los yucatecos, tomar en cuenta sus hábitos, tradiciones sin nunca alejarse de los principios de justica, de la protección al débil y sin dejarse dominar por los grandes.

Fomentar la agricultura y el comercio.

Terminar la Guerra de Castas a la mayor brevedad

Facultades militares

Vigilancia de la Hacienda y Economía

Decir a los yucatecos que son los hijos más queridos por los emperadores

Arreglo de la administración de justicia según la nueva ley 


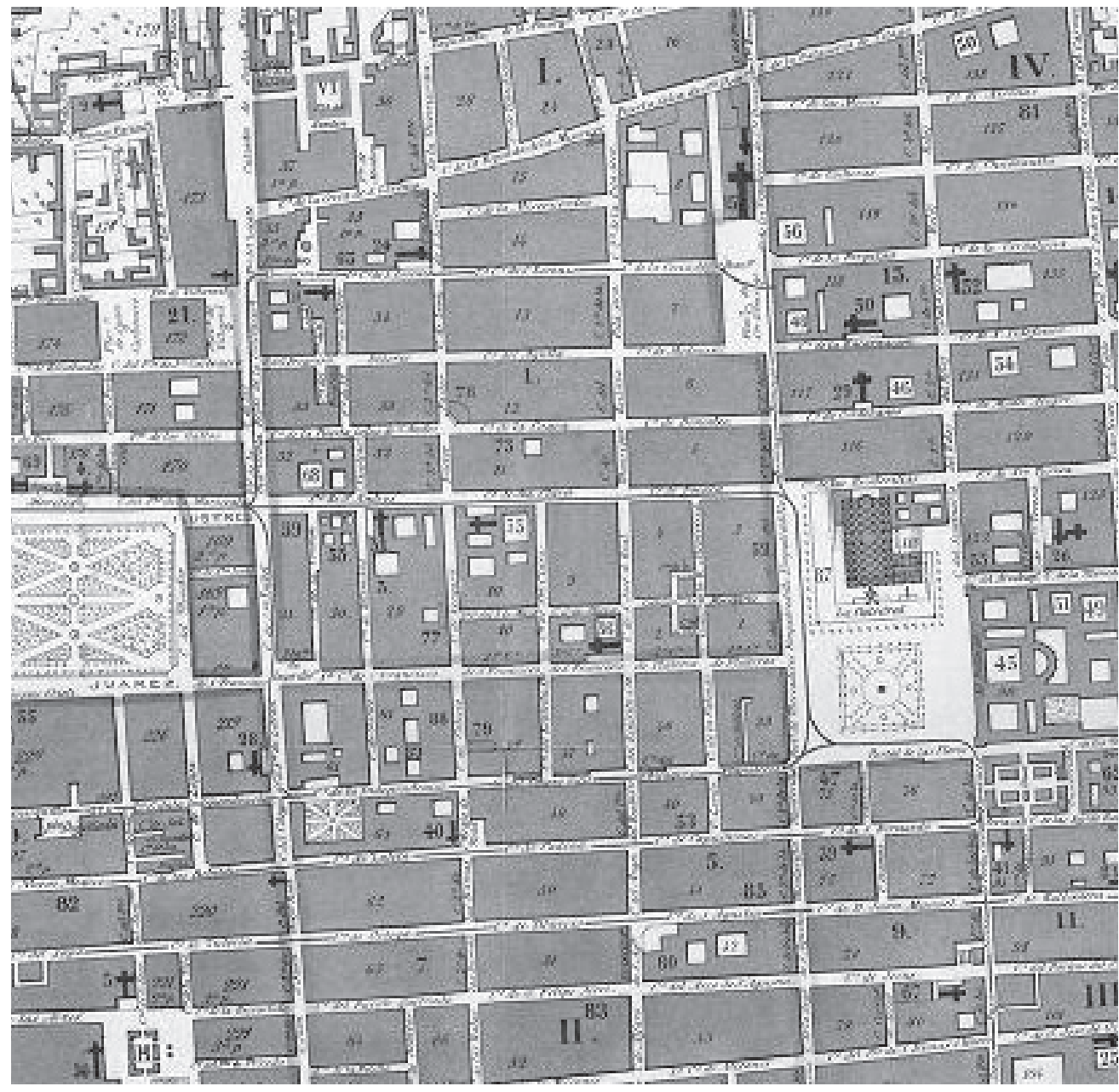

Figura 9.

Ciudad de México en 1875. Detalle.

Fuente:

http://www.

davidrumsey.com/

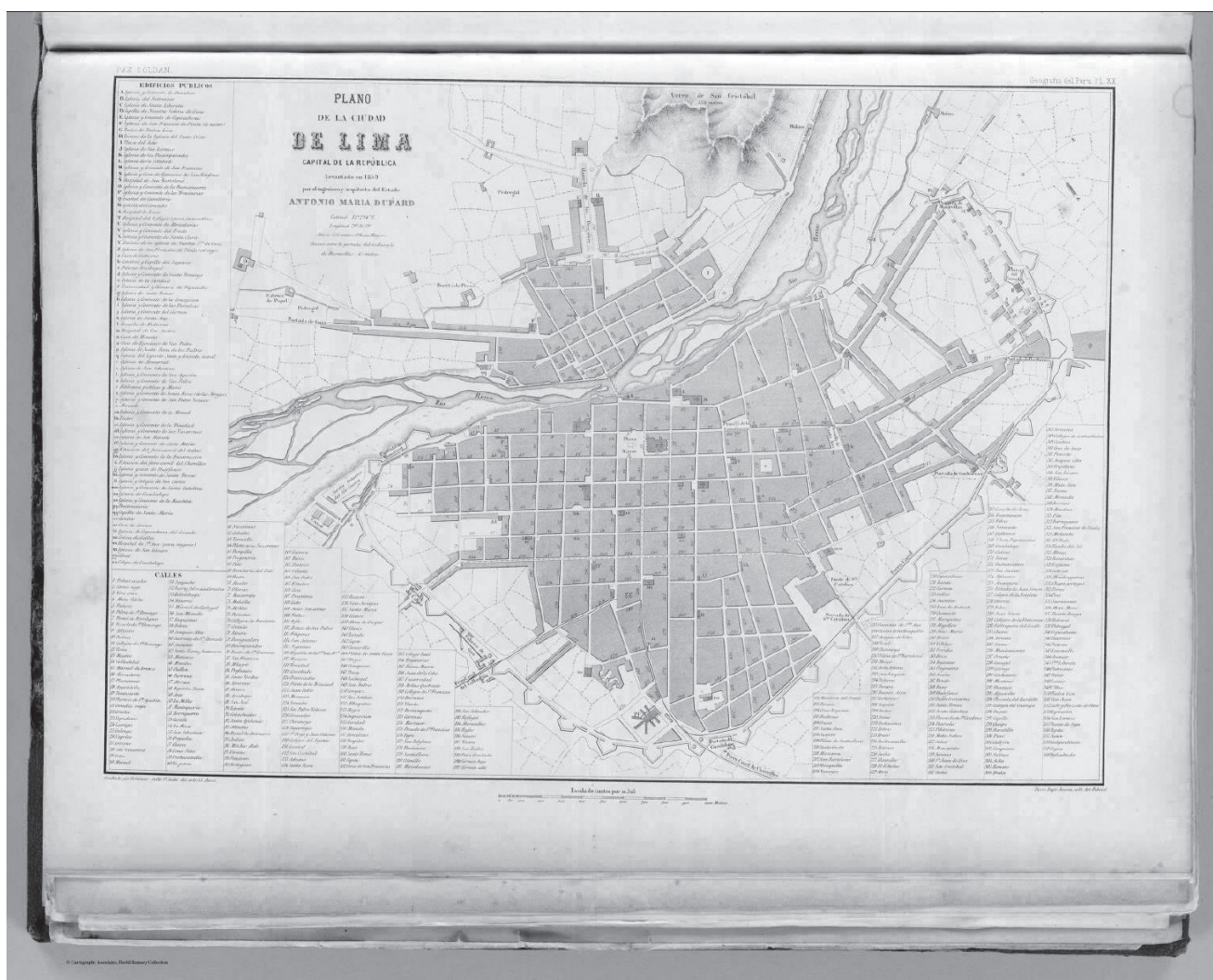

Figura10.

Lima. Plano de la ciudad en 1865.

Fuente:

http://www.

davidrumsey.com/ 
Figura 11.

Ciudad de Mérida.

Panorámica.

Fuente:

Desiree Charnay (1887).

Ancient Cities of the

New World, p. 267.

Figura 12.

Ciudad de Mérida.

Calle con viviendas.

Fuente:

Desiree Charnay (1887). Ancient Cities of the New World, p. 285.

Figura 13.

Ciudad de Mérida.

Palacio Municipal.

Fuente:

Desiree Charnay (1887).

Ancient Cities of the

New World, p. 262.
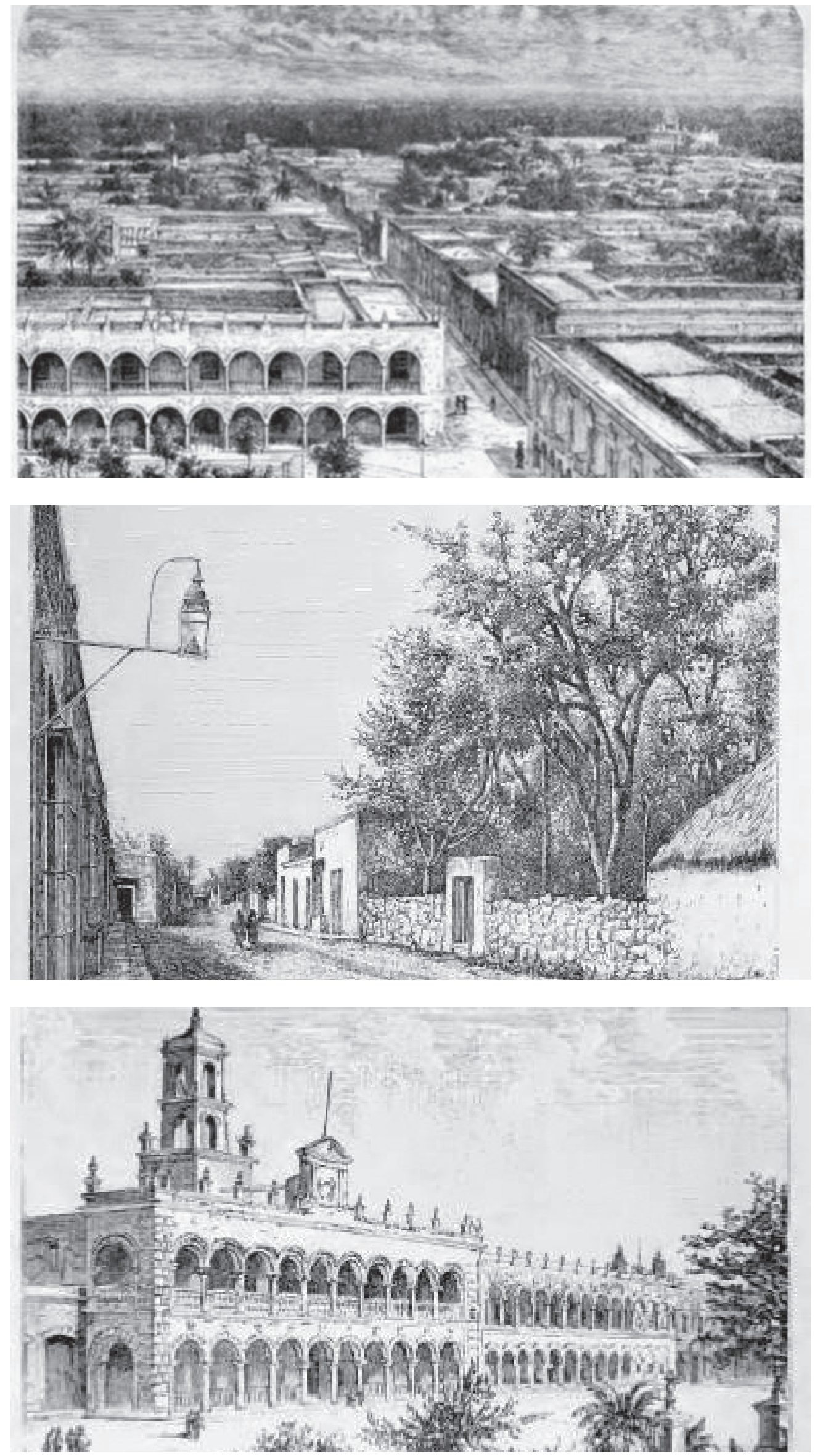
Siempre, en cuanto sea posible, hacer que la península se baste a sí misma y no cueste más de lo necesario y de las mesadas Reunirle Tabasco y Chiapas

Encargarse de las ruinas de estos cinco departamentos haciendo los gastos consiguientes a su mantenimiento y reparación Comisión nombrada al efecto

Conservadores en los lugares de las ruinas Museo en Mérida

Carta antigua de la península con sus calzadas y ciudades

Atlas de las ruinas

Modelo de ellas para el museo de Mérida y el nacional de México

Las instrucciones generales podrá redactarlas en palacio el señor Castro [amanuense del palacio].

Las secretas redactaría el señor Salazar para los ocho comisarios (Iturriaga, 2011, pp. 17-18).

Para la realización de muchas de estas acciones que hacían parte del plan modernizador, el paso inicial fue el conocimiento del territorio, tanto en relación a su estado físico como al económico. Por ello, en cuestión de dos meses, la Comisión científica designada levantó y dibujó el plano de la ciudad y se iniciaron las labores complementarias a este como los estudios y el análisis para la regularización y modificación a la ley de terrenos baldíos, un censo, el establecimiento y reglamentación de la dirección de caminos publicada en 1866, así como la administración general de rentas del mismo año ${ }^{12}$.

El trabajo lo realizaron doce ingenieros entre los que se encontraban Mauricio Von Hippel, José Iglesias, Carlos Ramiro y Carlos Moya, un yucateco de ascendencia alemana Joaquín Hübbe y un naturalista, artista y geógrafo de nacionalidad alemana, Arturo Schott. A fines del año 1864, Von Hippel partió hacia Europa con la comisión de traer colonos alemanes agricultores a Yucatán y llevando consigo el plano de la ciudad de Mérida para ser litografiado allí (Espadas, 1991, p. 3) ${ }^{13}$.

El ferrocarril de Progreso [a Mérida] será de la mayor importancia; prueba de ese espíritu de empresa que distingue a los yucatecos y que tanto estimo en ellos como en los Vera Cruzanos, pues es seguramente la mejora garantía para un venturoso porvenir

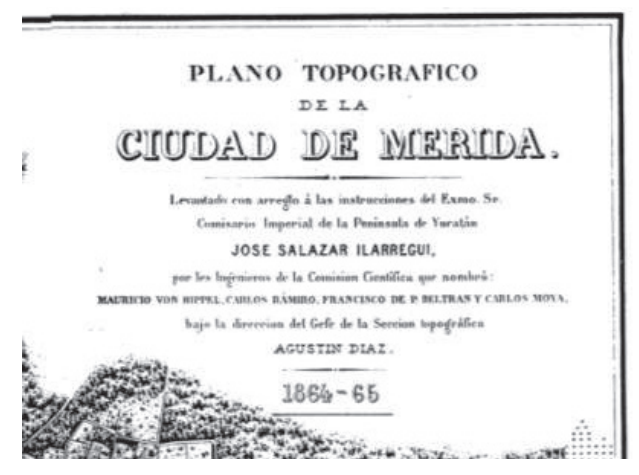

(...) Todas mis simpatías han sido y quedan siempre para Yucatán y tomo vivísima parte en sus adelantos y propiedad (...) Los yucatecos son verdaderamente los príncipes de avanzada en México, vivaces como los andaluces, joviales con un toque de caballerosidad, y dotados de un sentido mercantil como los habaneros. Son monárquicos por naturaleza, no se preocupan gran cosa de sentimentalismos demócratas, y saben combinar la poesía con lo prosaico (...) Entre los oficiales de Yucatán podías escoger apuestos oficiales de ordenanza [le sugiere a Maximiliano]. En las escuelas, los niños leen poemas de poetas yucatecos. Hay mucha inspiración poética (...) Arrojaron una paloma dentro de mi coche a cuyas patas estaban atados poemas con adornos tricolores (...) (Iturriaga, 2011, p.11).

Así, menos de un año después del arribo del comisario ejidal y sus múltiples encomiendas, llegó al puerto de Sisal la emperatriz Carlota, cuyos motivos eran hasta cierto punto inciertos. El emperador había programado su visita pero por motivos políticos más apremiantes tuvo que cancelar su viaje, confiriéndole a su esposa todas las licencias para representarlo. Así, la emperatriz reconoció una ciudad un tanto distinta y la percepción en relación a los yucatecos fue más que afortunada, aparentemente todo estaba dado para que Yucatán y Mérida fueran el centro político, cultural y de servicios de la región idealizado a la distancia por Napoleón 111.

La ciudad representada. El plano, las densidades, calles y edificios importantes

Compositiva y formalmente, el plano es simple, equilibrado y aunque emplea un lenguaje fácil de comprender es esencialmente técnico,
Figura 14.

Plano de Mérida

1864-1865. Detalle

de la información contenida en la esquina superior derecha.

Reprografía:

Gladys N. Arana

López, 2012.

12. Estos documentos se encontraron en la Universidad de Texas en la biblioteca Arlington, entre e acervo de la familia Arrigunaga. El catálogo se puede consultar en su modalidad virtual en la dirección http://www.lib.utexas. edu/taro/utarl/00178/arl00178.html\#a0, consultado el 15 de diciembre 2009.

13. Este hecho permite reflexionar en torno a lo que se deseaba del plano y la calidad deseada en él. En primer lugar, por el hecho de que ya existían excelentes talleres de grabado en la ciudad de México desde muchos años antes, sobre todo el establecido en la academia de San Carlos en donde Mariano Castro, bajo la supervisión de Don Ignacio Serrano, había grabado la litografía de un dibujo alegórico para la invitación de los festejos de septiembre. También se fundaron talleres públicos como el de Rocha y Fournier en donde se hicieron las litografías de la Historia de México, de Veytia publicada en 1836, así como aquellas publicadas en los primeros periódicos ilustrados: $E l$ Mosaico Mexicano (18371840) y el Recreo de las Familias (1838). Ya para el año de 1838 se estableció en México una litografía venida directamente de París, donde se hicieron obras mucho más perfectas que las anteriores. Muchas fueron las obras litografiadas con perfección desde aquel entonces, destacándose la colección publicada por Decaen México y sus alrededores (1855-1856).

Sin embargo en Mérida, hasta mediados del siglo XIX no había taller litográfico; las ilustraciones del famoso Registro Yucateco (4vols., 1845-1846) se realizaron en la Habana, Cuba y estaban firmadas "Lit. de la R.S.E. c. de Cuba 22, Habana", mientras que las del curioso folleto "Vida de Fr.

Manuel Martínez", realizado por D. Crescencio Carrillo 


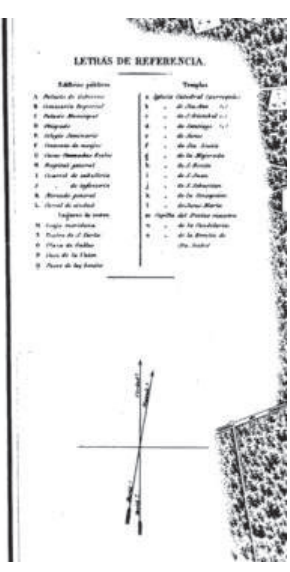

Figura 15. Plano de Mérida 1864-1865. Detalle de la información contenida en la esquina inferior derecha.

Reprografía:

Gladys N. Arana

López, 2012.

Figura 16. Plano de Mérida 1864-1865. Detalle de la información contenida en la esquina inferior izquierda.

Reprografía:

Gladys N. Arana López, 2012.

Figura 17. Plano de Mérida 18641865. Detalle de la zona de la ciudadela de San Benito. Reprografía: Gladys N. Arana López, 2012.

y Ancona en 1883 , parece que sí fue realizada en Mérida, pero carecen de firma. (Toussaint, 1934, pp. 5-10).

14. En caso de tomarse en cuenta esa fecha. la aseveración del Arq. Aercel Espadas sobre la fecha en que Von Hippel viajó a Francia a litografiar el documento, (noviembre de 1864) sería incorrecta, a menos que la indicación de la desviación magnética se haya realizado estando ya la información en tierras europeas. Por otra parte, el mismo arquitecto indica que el proyecto de la nomenclatura de la ciudad de Mérida se realizó en el trayecto del viaje, lo que implicaría otra modificación o adición al documento. Al respecto, plantearía algunas preguntas: ¿Realmente el proyecto de la nomenclatura se hizo
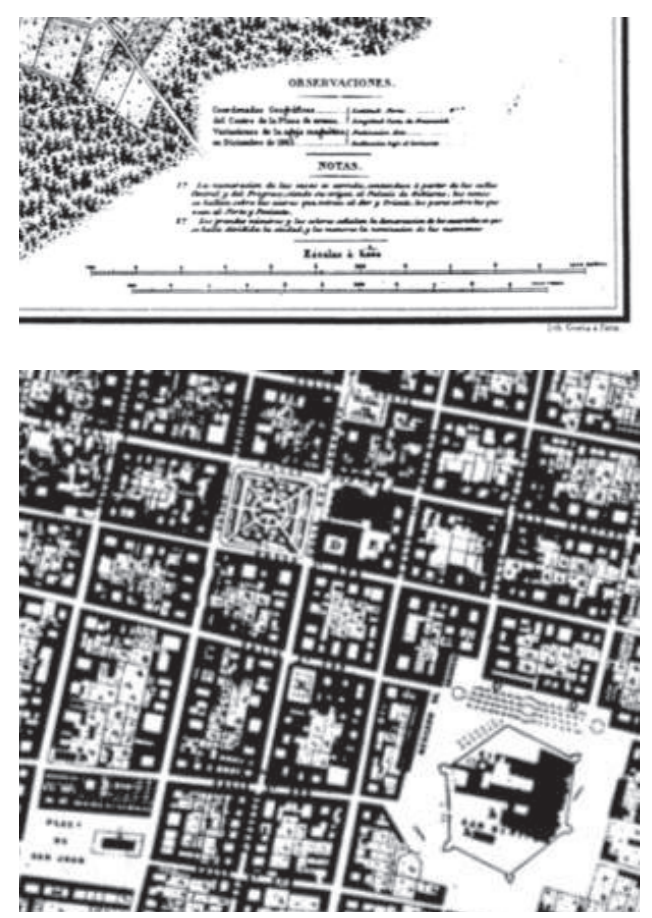

la información escrita en él está distribuida en tres secciones: en un primer grupo localizado en la esquina superior derecha del documento se encuentran los datos generales del plano, en donde con distinta fuente y tamaño tipográfico, se enfatiza el título del documento, el nombre de Salazar como comisario y el año de elaboración del trabajo; también se pueden leer los nombres de los integrantes de la comisión científica.

En la esquina inferior derecha, en el segundo grupo de información se encuentra el apartado de observaciones, en donde se incluyen las coordenadas geográficas referenciadas al centro de la plaza de armas y las variaciones de la aguja magnética a diciembre de $1865^{14}$. También hay un apartado de notas donde se enuncia el criterio y origen de la numeración de las casas y la explicación correspondiente a los números y los colores referidos a la demarcación de los cuarteles y las manzanas, por último se encuentran una escala escrita y dos gráficas ${ }^{15}$.

El tercer grupo de información está en la esquina inferior izquierda del documento en donde podemos ver la indicación gráfica del norte geográfico y la desviación de la traza, así como una relación de Letras de referencia divididas en tres grandes grupos: edificios públicos, lugares de recreo y templos.

La lectura del plano topográfico posibilita conocer la ciudad en un tiempo especifico y con bastante precisión. En él, al tenerse la panorámica de todo el emplazamiento, se pueden observar las densidades constructivas, las calles principales, la vegetación, así como todos y cada uno de los edificios existentes en la época y algunas especificidades arquitectónicas, tales como la composición básica de los edificios, las proporciones de sus patios, su relación con la calle y el vínculo con las otras construcciones, por mencionar algunas.

En relación a la densidad constructiva se observa que esta es mayor sobre los dos ejes perpendiculares que atravesaban completamente la ciudad. Uno de ellos correspondiente al camino prehispánico o sac-bé maya, tomado como referencia en el momento fundacional de la ciudad española y a la vereda del cual se había establecido la catedral y el obispado -llamada calle del Progreso-; estaba orientado norte-sur, mientras que la otra calle -orientada de oriente a ponientepasaba al frente de la casa del otrora fundador de la ciudad, Francisco de Montejo. Así se demarcaba un moderno cardo y decumanos y se enfatizaba la traza cartesiana del emplazamiento.

Otras dos calles atravesaban completamente la ciudad en el sentido norte-sur y una más con orientación este-oeste, conformando así una red de caminos que vinculaban totalmente a la ciudad con las poblaciones circunvecinas o bien con centros poblacionales significativos por distintas circunstancias, como lo era el caso de Sisal, el puerto comercial más importante de la época. Complementariamente a estas vialidades, otros diez caminos llegaban a Mérida, algunos de los cuales están simplemente indicados, mientras que otros sí están especificados por sus nombres, tales como el camino a ltzimná, a Catalina, a Kinchil y a Campeche, por medio de este último se comunicaba la ciudad de Mérida con el resto de México ${ }^{16}$.

La nomenclatura implantada por el segundo imperio representada en este plano, se basó en el sistema cartesiano, siendo su origen la esquina noreste de la plaza. Las calles en sentido norte-sur y paralelas a la llamada calle del Progreso se numeraban ordinalmente conforme se alejaban de él, a partir de la primera con el nombre del punto cardinal del sentido en que se alejaban de dicho eje. Se aplica el mismo criterio para las calles orientadas este-oeste (Espadas, 1991, p. 7).

Por otra parte, es notorio que en las áreas periféricas o cercanas a los barrios aún no se encontraban construcciones consolidadas, sino por lo contrario, tal pareciera que estos cen- 

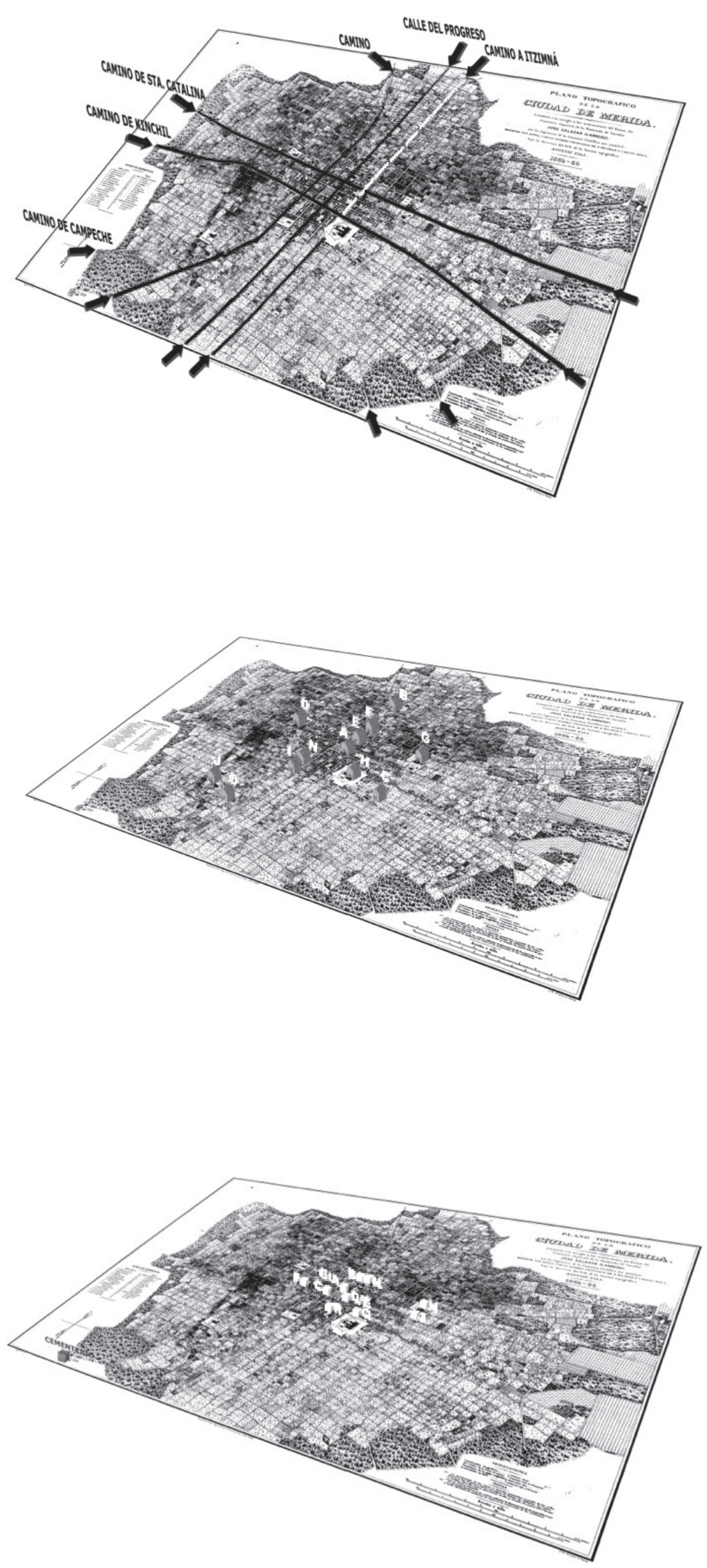

en el momento de llevar el levantamiento a Francia? ¿José Salazar en su calidad de comisario Imperial, no debería de estar a cargo y autorizar la misma, siendo meticuloso y conocedor de su trabajo? Y en ese sentido, ¿cabría la posibilidad de que el plano no tuviera como objetivo real coadyuvar en la implantación de la nueva nomenclatura?

15. El documento con el que se trabajó procedente de la mapoteca Orozco y Berra no tiene colores, se infiere que por la fecha del plano localizado en la biblioteca nacional de Francia, este podría tener los colores originales.

16. Fueron dos asentamientos, el barrio de Santiago y el de Santa Catalina, en donde los naturales vivían durante el período vierrinal, ya que Francisco de Montejo había respetado esta condición al momento de la fundación de la ciudad de Mérida, sin embargo este último barrio desapareció por una plaga y un incendio, sin que haya un registro preciso de este hecho. Por ello y ya que el asentamiento se menciona en el plano, se infiere que para la fecha de elaboración de este aún no había sucumbido el barrio ante las catástrofes mencionadas.

Figura 18.

Plano de Mérida 18641865. Caminos que llegaban a la ciudad y calles principales. Fuente:

Waldir Hernández y Gladys Arana, 2012.

Figura 19.

Plano de Mérida 1864-1865. Iglesias en la ciudad.

Fuente:

Waldir Hernández y Gladys Arana, 2012.

Figura 20.

Plano de Mérida 18641865. Equipamiento.

Fuente:

Waldir Hernández y Gladys Arana, 2012. 

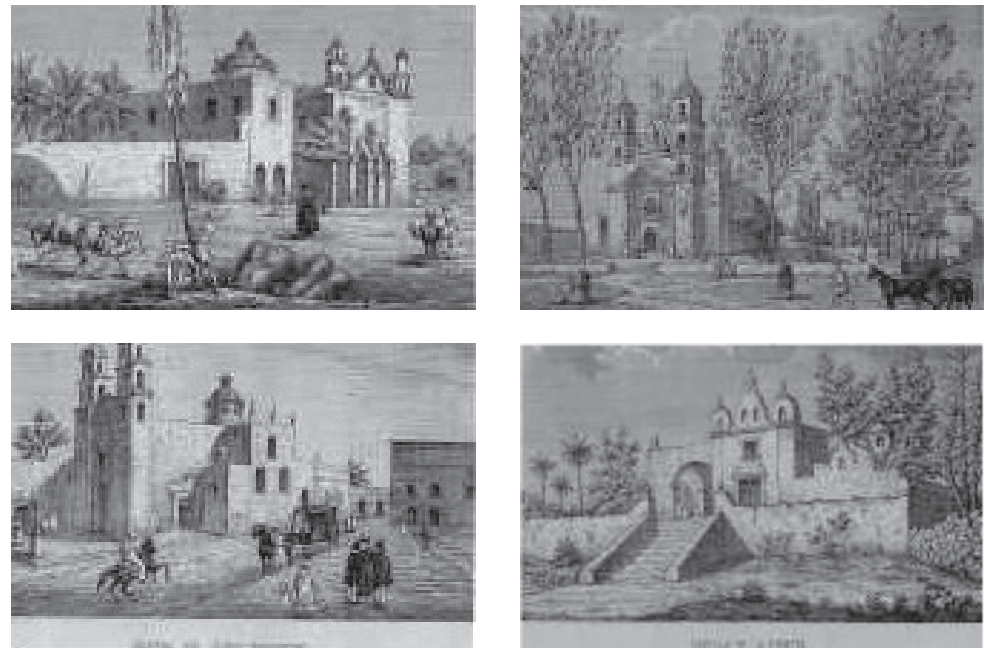

Figuras 21, 22, 23 y 24. lglesias en Mérida.

Mejorada, San Cristóbal, el Jesús y la Ermita de Santa lsabel. Fuente:

Michel Antochiw (1992). Mérida y su gente antes de la fotografía. Reprografía: Gladys N. Arana, 2012.

17. En ninguno de los casos se graficó el segundo espacio característico de la vivienda vernácula maya, siendo este el espacio en donde se cocinaba, quizás por estar construida con materiales incluso más perennes que aquellos de la casa maya principal. tros urbanos sirvieron como contenedores de la expansión de la ciudad. De igual forma y con la intención de controlar y organizar la ciudad, esta fue dividida en cuatro cuarteles y cinco barrios. Los cuarteles se localizaron en el área central de la ciudad, comprendiendo en total trece manzanas de norte a sur y diez de oriente a poniente. Las iglesias de los barrios, con excepción de San Sebastián, se encontraban en los bordes exteriores de los cuarteles (Espadas, 1991, p. 7).

Obviamente no hay señalización alguna a la vivienda en las letras de referencia contenidas en el plano, sin embargo se guardó cuidado en su representación. Cada una de las casas construidas en mampostería estaban representadas con la planta de conjunto correspondiente, sin importar lo sinuoso de su configuración, mientras que las vernáculas, localizadas en la periferia de la ciudad consolidada, estaban dibujadas en el chaflán de las esquinas, en lotes mucho más grandes y menos definidos, por medio de su característica planta absidal ${ }^{17}$. Todos los lotes, sin importar la tipología de la casa o de su consolidación, estaba definido en el gráfico, aunque no necesariamente estas delimitaciones eran físicas per sé.

Específicamente los emplazamientos señalados en el plano eran:

a. Los públicos. Entre ellos se encontraban el palacio de gobierno, la comisaría y el palacio municipal, el obispado, el colegio seminario, -cuya construcción se inició en 1618- el convento de monjas, las casas llamadas reales, el hospital general, el cuartel de caballería y el de infantería; el mercado general y la cárcel de la ciudad, en pocas palabras y de manera general, parte del equipamiento con el que contaba la ciudad y las instituciones más re- presentativas para la impartición de justicia, educación, seguridad y salud,

b. Lugares de recreo. ejemplos de ello son la lonja meridana, el teatro San Carlos, -cuya obra fue concluida en 1831 estando a cargo del arquitecto guatemalteco Manuel Cea Gómez- la plaza de gallos, la casa de la Unión y el Paseo de las Bonitas, sin marcar diferencia entre los públicos y los privados, abierto o cerrado. Esto pareciera evidenciar la intención de hacer de Mérida una ciudad incluyente sin diferenciación de clases sociales.

c. Los templos. Habría que mencionar en primer lugar, las parroquias como Catedral, Santa Ana, San Cristóbal y Santiago y posteriormente, las iglesias como el Jesús -construido a finales del siglo XVIl con ayuda de los habitantes de la ciudad- (Ancona, 1991, p. 19), Santa Lucía, Mejorada, San Benito, San Sebastián, de la Concepción, Jesús María, el Divino Maestro, la Candelaria y la ermita de Santa lsabel.

Todo esto es de relevancia, ya que en conjunto con la serie litográfica realizada sobre las iglesias, el plano proporcionaba un panorama fiel de esta institución en la ciudad y si bien ya se le había devuelto su función a los curas y párrocos, se había suspendido la nacionalización de capitales en manos de la iglesia y se había facultado a los clérigos para realizar actos y prácticas religiosas en lugares públicos sin permiso de la autoridad política; era necesario saber qué había y dónde estaba, por lo que quizás estos documentos sustituyeron al registro realizado en otros Estados sobre el proceso de desamortización de las propiedades rústicas y urbanas de las corporaciones eclesiásticas y civiles a raíz de la ley del 25 de junio de 1856.

\section{¿Omisiones voluntarias? \\ Lo no representado}

De lo más simple a lo más complejo, muchas son las cosas que en aquel entonces existían en la ciudad y que no se dibujaron en el plano. ¿Por qué se omitieron? ¿Por qué no se querían referenciar? ¿Se pretendía demoler estas preexistencias?, ¿o es que acaso se quería representar una ciudad ideal sin algunas de sus condicionantes naturales y de sus precedentes arquitectónicos?

Uno de los ejemplos que podríamos mencionar es la llamada Casa Mata o polvorín. Su omisión 


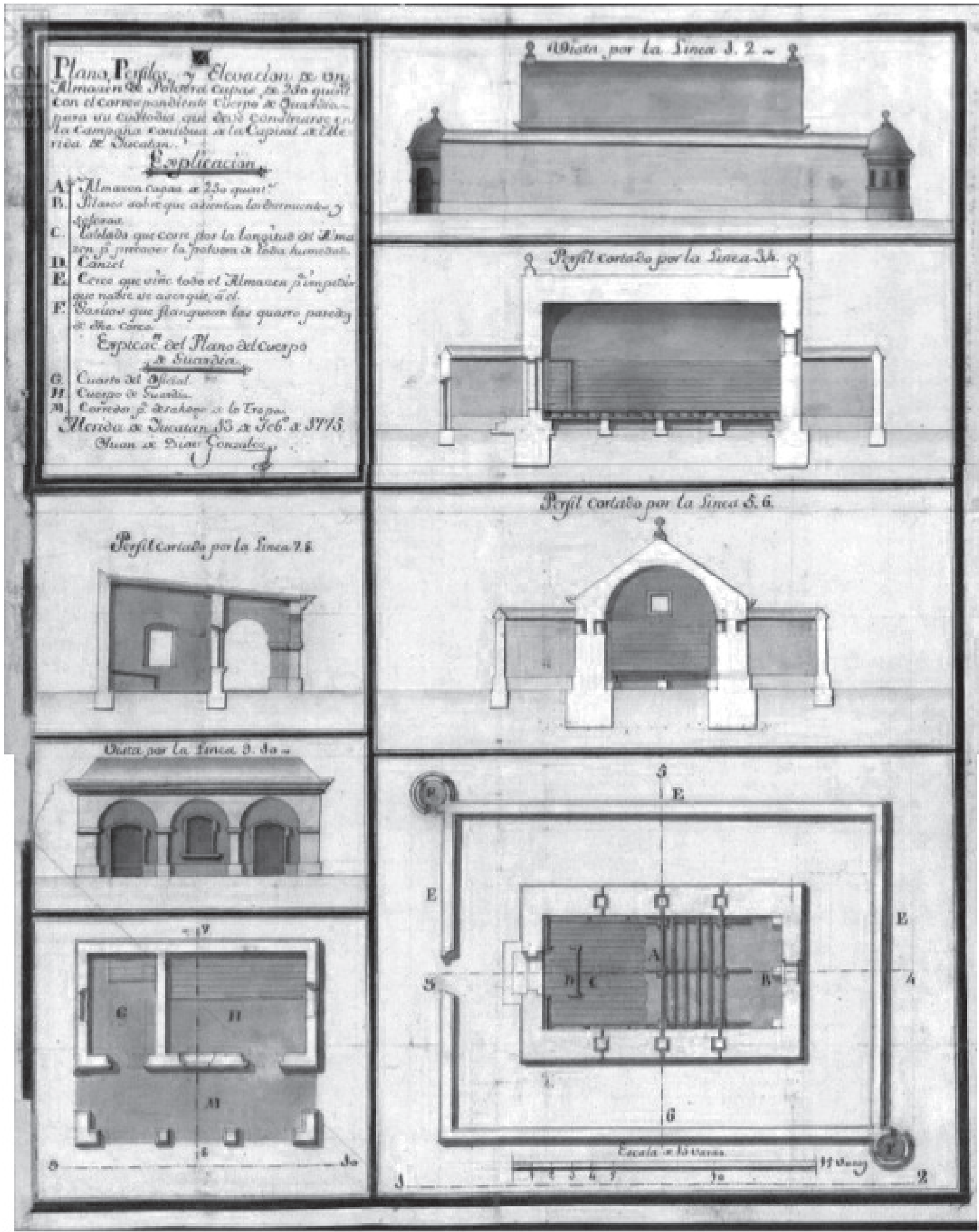




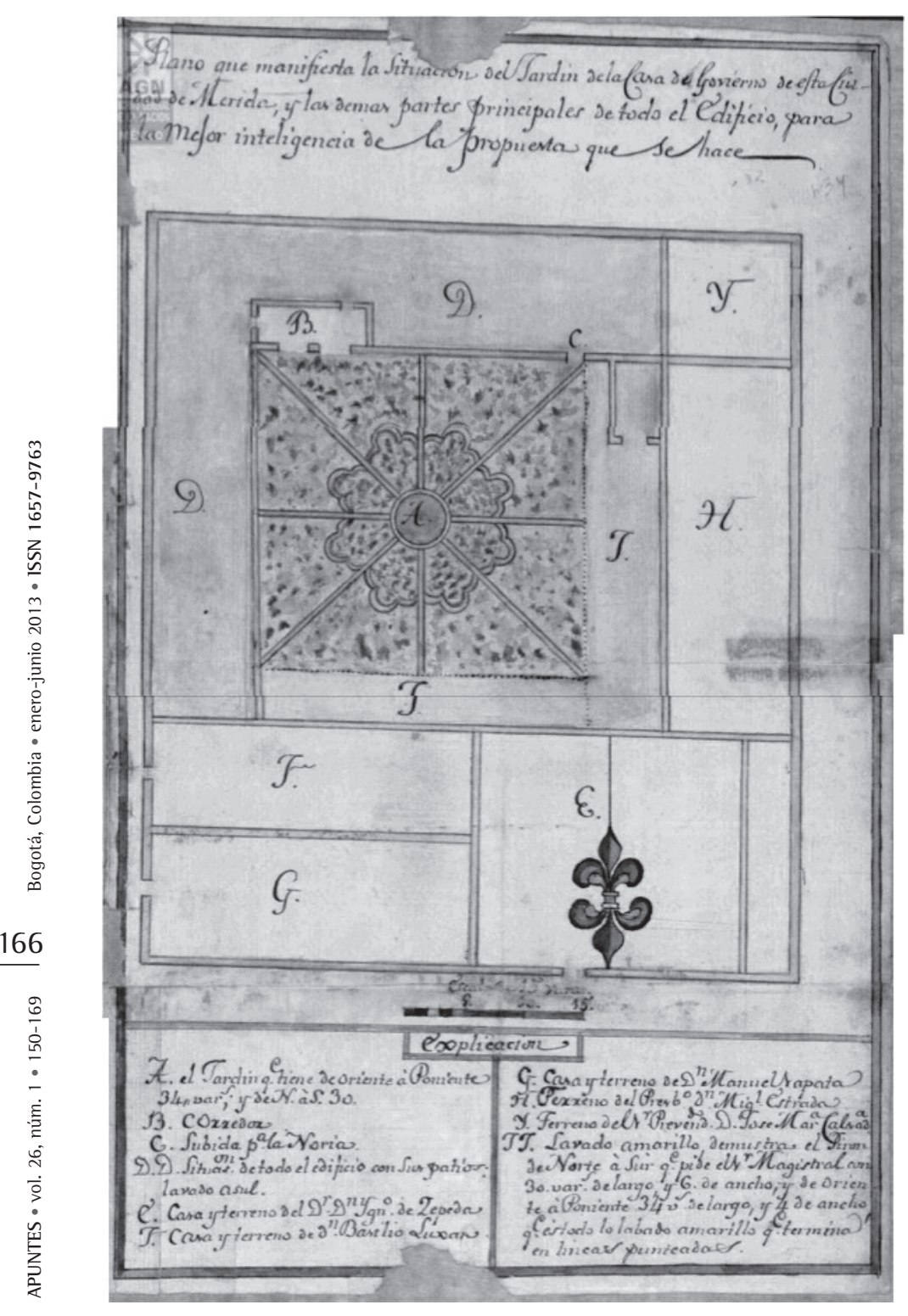

Figuras 25, 26, 27. Edificios públicos en Mérida. Casa de pólvora 1775 (registro 4372),

Arzobispado 1805 (registro 3452), jardín de la casa de gobierno 1812 (registro 2686).

Fuente: AGN.

18. En aquel entonces existían unos árboles conocidos como ramones, predecesores de los laureles sembrados en 1870. es importante y puede dar pie a múltiples interpretaciones, ya que estando en medio de un conflicto bélico y teniendo las intenciones de concluirlo, las instituciones militares y de control social estaban representadas en el plano demostrando así la capacidad del Estado de afrontar sus problemas, por lo que si bien esta edificación ya no almacenaba todo el arsenal, sí era representativa y significativa como parte del lenguaje del poder de la clase gobernante frente a las masas. Lo mismo se podría decir de los arcos que preexistían en aquel entonces, entre los que estaban los de San Juan, San Cristóbal y el del Dragones, edificaciones que otrora habian protegido a la ciudad.

Hay otro tipo de faltante en la representación de la ciudad de Mérida, sobre todo ante las intenciones de modernizarla. Y es que dibujar la localización de todos los cenotes mas importantes con posibilidad de ser explotados acuíferamente, hubiera sido un factor coadyuvante al momento de decidir la localización de los pozos artesianos y de la noria, cosa que en apariencia no ocurrió. Por otra parte, a pesar de haber sido considerado como esencial en el desarrollo de Mérida e incluso recomendado por la emperatriz Carlota en su visita, las escuelas no se encontraron en la mancha urbana retratada y mucho menos fueron enunciadas. Por último, si bien se dibujó la vegetación correspondiente al monte periférico a la ciudad, ninguno de los árboles de ornato se representaron, reduciéndose estos al ejercicio de graficar sus correspondientes troncos, hecho que se puede observar con claridad en el Paseo de las Bonitas y en la Plaza de $\operatorname{Armas}^{18}$.

Por último, en el plano no existe referencia alguna al imperio, hecho que causa extrañeza sobre todo ante el hecho de que Maximiliano de Habsburgo tenía el distintivo de su imperio en todas partes, desde el uniforme de sus soldados, hasta su papelería personal. Tampoco hay evocación alguna a otros proyectos a desarrollarse, tales como el del telégrafo, el ferrocarril y el parque botánico.

\section{Lo representado y no enunciado}

También representado mas no enunciado estaba el cementerio, construido en 1821 y administrado por la iglesia hasta 1859. Esta ausencia causa curiosidad, ya que el cementerio era un emplazamiento congruente con la concepción de una ciudad moderna decimonónica. En un caso 
similar se encuentran las quintas Miraflores y San Jacinto, cuya representación detallada quizás se debió a la intención de repartir tierras fértiles y próximas a los emplazamientos urbanos entre los futuros inmigrantes alemanes. Otro edificio de relevancia histórica y arquitectónica representado en el plano y no enunciado fue la residencia del comisario imperial, que había sido aduana y en diversos momentos de su historia, un importante centro de estudio (Ancona, 1991, p. 22).

Si bien el plano permite conocer la ciudad de aquel entonces, algunos elementos no presentan coherencia al vincularlos o confrontados con otros documentos, con acciones implementadas en otras ciudades e ideales urbano-arquitectónicos de la época. Por ello, considerando que Maximiliano era fiel seguidor de las propuestas urbanas del barón Haussman y que a partir de ellas, se realizaron algunas de las obras más representativas del urbanismo decimonónico mexicano, ¿por qué si se deseaba que Mérida fuera la capital política de la región no se planteó un desarrollo urbano de relevancia en la ciudad? o al menos, ¿por qué no se hizo de manera explicita?

Partiendo del supuesto de que el plano encargado por José Salazar llarregui, más allá de ser la representación de lo existente, tuvo como objetivo ser la base y fundamento para el desarrollo de un proyecto urbano arquitectónico más ambicioso, se puede plantear que la actual calle 56, paralela a la calle del Progreso, realmente sería un paseo que iniciando en la alameda y en el llamado Paseo de las Bonitas -mencionado en el plano-, se desarrollaría hacia el norte y concluiría en la bifurcación del camino a ltzimná. Se puede observar que la mencionada calle es más ancha ${ }^{19}$ que las calles circunvecinas y que además, midiendo la secuencia de chaflanes localizados en esta vialidad -que en su conjunto conforman pequeñas glorietas- estos están separados por quinientos metros, lo que coincide con la distancia entre monumentos en el Paseo de la Reforma en la ciudad de México, proyecto del gobierno imperial. Complementariamente, en esta calle se localizaron las principales casas comerciales de finales del siglo XIX y si bien fueron fincadas por alemanes, fungieron como las casas comerciales francesas que predominaron en ciudades como México y Veracruz.

Por último, el análisis del registro de fincas urbanas realizado en el año 1903, indica que las casas más ostentosas y ricas de la ciudad de Mé-

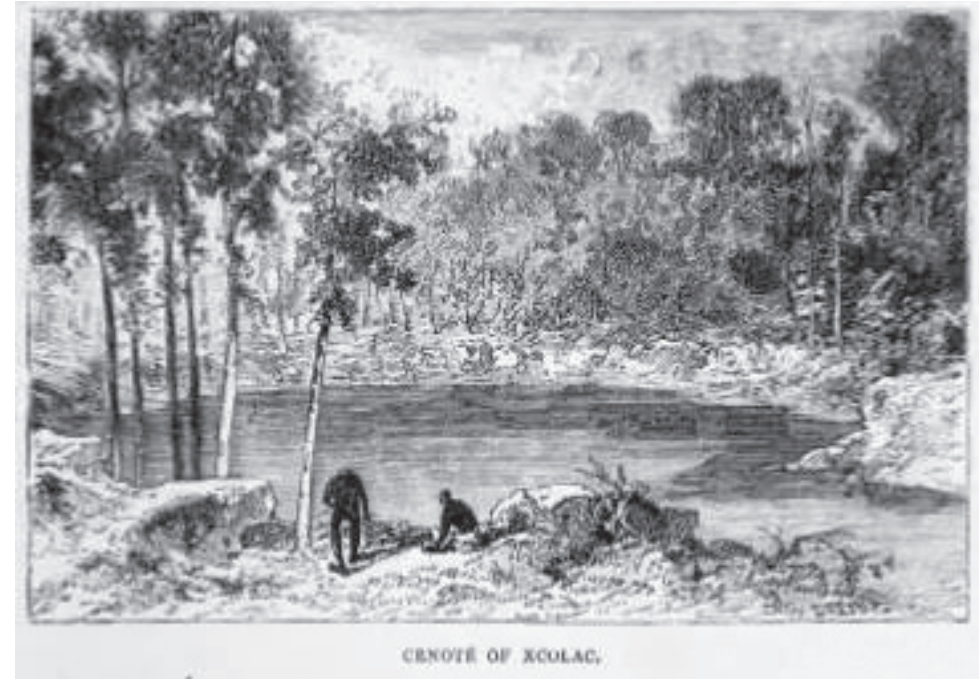

rida se encontraban en esa vialidad perteneciendo a familias de gran tradición social en la región. No está de más decir que la calle 56 se encuentra a cien metros de la que se volvería el Paseo de Montejo, avenida insigne del porfirismo, que sin embargo se considera como una propuesta aislada y fuera de contexto urbano, entonces ¿será que el proyecto del Paseo de Montejo no fue realmente la primera iniciativa? Quizás lo efímero del segundo imperio no permitió la realización de este proyecto urbano, que integraría diversas partes de la ciudad de forma congruente con los planteamientos ideales del urbanismo francés.

\section{Conclusiones}

La intención de analizar y cuestionar al plano topográfico de la ciudad de Mérida realizado durante el efímero imperio de Maximiliano de Habsburgo entre 1864 y 1867, ha permitido aproximarse a la relación entre el documento y el territorio que representa. Algunas de las preguntas formuladas en un principio en relación al territorio, a la utilidad del documento y sus objetivos se respondieron, si bien no de manera directa o explícita, sí mediante la lectura de otros elementos y documentos que habían sido obviados previamente.

En primer lugar el conocimiento de las circunstancias técnicas y temporales de su ejecución, así como también del sujeto que lo encargó y supervisó su elaboración, posibilitó contextualizar tanto al documento como al territorio mismo en un ejercicio dialógico. Por otra parte, lo representado, lo ausente y lo implícito, adquirió una nueva
Figura 28.

Entorno natural. Cenote.

Fuente:

Desiree Charnay (1887). Ancient Cities of the

New World, p. 317.

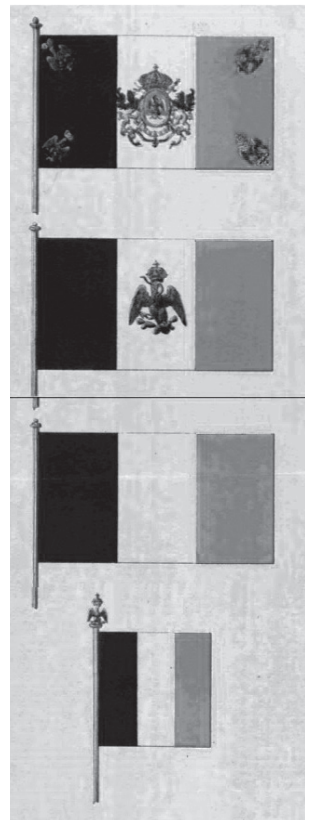

Figura 29.

Banderas mexicanas (registro 3477.1), 1864. Fuente: AGN

19. Aproximación realizada por medios digitales. 


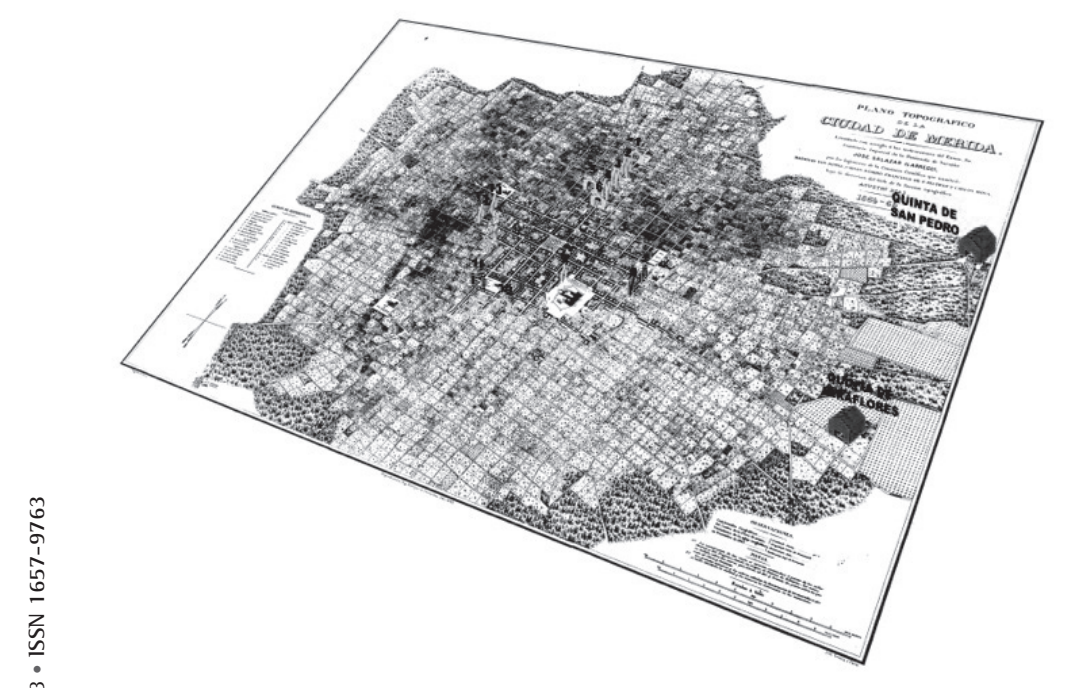

168

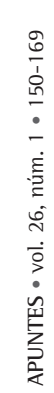

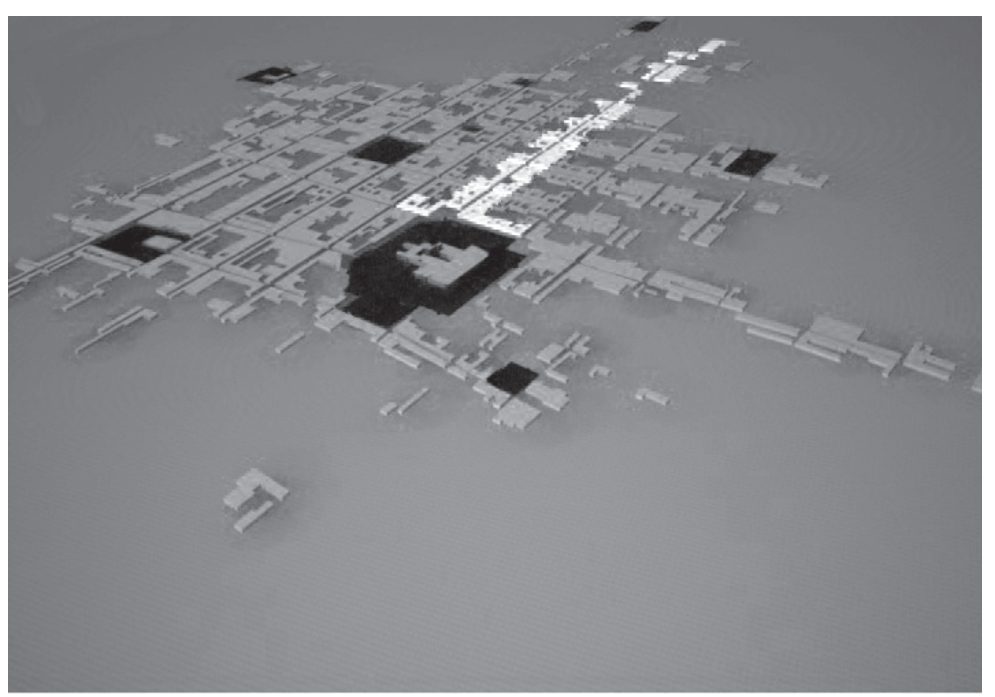

Figura 30.

Plano de Mérida 18641865. Localización de las fincas cercanas a la mancha urbana y los arcos construidos. Fuente:

Waldir Hernández y Gladys Arana, 2012.

Figura 31.

Plano de Mérida 18641865. Levantamiento tridimensional de la ciudad de Mérida enfatizando la calle 54. Fuente:

Waldir Hernández y Gladys Arana, 2012. dimensión al expresarse bajo el tamiz de las verdaderas intenciones y de los significados ocultos.

Por último, se puede considerar que el plano tuvo como objetivo primario el conocimiento de la ciudad, pero también se podría decir al mismo tiempo que fue la herramienta empleada para plantear una serie de acciones modernizadoras que incluían la desaparición de algunos elementos, la negación de otros y la edificación de algunos más. Para ello, la ciudad, al ser representada en el plano, fue deconstruida gráficamente separando pasado de presente en aras de un porvenir mucho más promisorio.

Ya fuere como mecanismo coadyuvante para el control económico, político y social o como base para la propuesta de una ciudad en un imperio ideal, el plano topográfico de la ciudad de Mérida es un documento en el que lo real, lo posible y lo ideal se amalgamaron. Al fin y al cabo, solo la capacidad política y económica, así como la voluntad social permitieron el desarrollo de la ciudad, unos años después en el albor del siglo XX.

\section{Referencias}

Ancona, R. (1991). Arquitectura jesuítica en Yucatán. Cuadernos de arquitectura, 4, 17-31.

Antochiw, M. (1992). Mérida y su gente antes de la fotografía. Mérida: CULTUR.

Antochiw, M., y Alonzo, R. (2010). Santiago Servián y el primer plano de la Ciudad de Mérida. En Hechos de Yucatán (pp. 173-178), Mérida: Gobierno del Estado de Yucatán-ICY.

Barbachano y Tarrazo, M. (1951). Vida, usos y hábitos de Yucatán al mediar el siglo XIX. Mérida: Maldonado Editores.

Cetina, J. (1984). Historia gráfica: Mérida de Yucatán 1542-1984. Mérida: Bassó.

Connolly, P. (2008). ¿El mapa es la ciudad? Nuevas miradas a la Forma y Levantado de la Ciudad de México 1628 de Juan Gómez de Trasmonte. Investigaciones geográficas, Boletín del Instituto de Geografía, 66, 116-134.

Durán-Merck, A. (2009). Villa Carlota: colonias alemanas en Yucatán, Mérida. Ecuador: 1CY, CEPSA.

Espadas, A. (1991). La Nomenclatura de Mérida, 1864-1877. Cuadernos de Arquitectura de Yucatán, 4, 1-16.

Espadas, A. (1993). La traza borbónica última virreinal, primera modernización. En El Azar y la Memoria (pp. 45-88), Mérida: APAUADY.

Espadas, A. (2011). Mérida sin M ni R., sin mitos ni romanticismos de su fundación hasta su segunda modernización. Cuadernos de Arquitectura de Yucatán, 23, Mérida, FAUADY.

Gahona, G. (2005). D. Bullebulle. Mérida: ICY.

Hiersemann, K. (1913). Catalogue Bibliotheca Mexicana. Leipzig: Hiersemann.

Iturriaga, J. (2011). Viaje a Yucatán. México: Summa.

Lapointe, M. (2008). Historia de Yucatán, siglos XIX-XX. Mérida: UADY.

Monroy, 1. (2003, septiembre-diciembre). La resistencia liberal y popular en la península yucateca durante el segundo imperio, 18651867. Cuicuilco, 10 (029), 1-23.

Peraza, M. (2009). Retrato de una utopía: el plano de la ciudad de Mérida de José Salazar llarregui (1864-1867). En C. Vargas y E. Ayala (Coords.) Arquitectura y Ciudad. Métodos 
Historiográficos: Análisis de Fuentes Gráficas (pp. 129-144 ), México, UAM-XOCH.

Pickenhayn, J. (2005). El nacimiento de una disciplina: la geografía en México. Siglos XV1 a XIX. Investigaciones Geográficas, 056, 166-168.

Quezada, S. (2011). Historia breve de Yucatán. México: FCE-COLMEX.

Reed, N. (1971). La guerra de castas de Yucatán, México: Era.
Suárez, V, (1977). Historia económica de Yucatán a través del siglo XIX. Mérida: UADY.

Toussaint, M. (1934). La litografía en México. México: Biblioteca Nacional.

Vega, R. (2003). El ensanche de Mérida durante el porfiriato. En Alonzo y Tello (Coord.). Evolución y estrategias del desarrollo urbano ambiental en la península de Yucatán, Mérida: UADY.

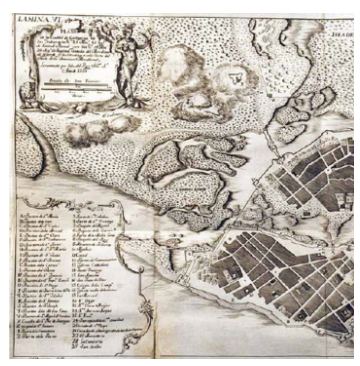

